\title{
The Pressure Equation in the Fast Diffusion Range
}

\section{Emmanuel Chasseigne and Juan Luis Vázquez}

We consider the following degenerate parabolic equation

$$
v_{t}=v \Delta v-\gamma|\nabla v|^{2} \quad \text { in } \quad \mathbb{R}^{N} \times(0, \infty),
$$

whose behaviour depends strongly on the parameter $\gamma$. While the range $\gamma<0$ is well understood, qualitative and analytical novelties appear for $\gamma>0$. Thus, the standard concepts of weak or viscosity solution do not produce uniqueness.

Here we show that for $\gamma>\max \{N / 2,1\}$ the initial value problem is well posed in a precisely defined setting: the solutions are chosen in a class $\mathcal{W}_{s}$ of local weak solutions with constant support; initial data can be any nonnegative measurable function $v_{0}$ (infinite values also accepted); uniqueness is only obtained using a special concept of initial trace, the $p$-trace with $p=-\gamma<0$, since the standard concepts of initial trace do not produce uniqueness.

Here are some additional properties: the solutions turn out to be classical for $t>0$, the support is constant in time, and not all of them can be obtained by the vanishing viscosity method. We also show that singular measures are not admissible as initial data, and study the asymptotic behaviour as $t \rightarrow \infty$.

\section{Introduction}

We study the following nonlinear degenerate parabolic equation:

$$
v_{t}=v \Delta v-\gamma|\nabla v|^{2} \quad \text { in } \quad Q=\mathbb{R}^{N} \times(0, \infty),
$$

where $\gamma$ is a real parameter. We look for nonnegative solutions of the Cauchy problem, not necessarily bounded. For $\gamma>0$ the problem is not well-posed in the usual classes of classical, weak or viscosity solutions.

2000 Mathematics Subject Classification: 35K55, 35K65.

Keywords: Pressure equation, fast diffusion; well-posed problem, non-uniqueness; measure as initial trace, optimal initial data. 
The purpose of this paper is to show that the Cauchy Problem (Equation (1.1) with initial data $v(x, 0)=v_{0}(x)$ for $\left.x \in \mathbb{R}^{N}\right)$ is well-posed with arbitrarily large initial data, more precisely in the class $\mathcal{L}^{+}$of all nonnegative measurable initial functions ( $v_{0}$ can be infinite on a general measurable set), if we understand the initial data in an unusual sense, namely as Borel $p$-traces with $p=-\gamma<0$. The result holds for $\gamma>N / 2$. It is remarkable that, while the class of data $\mathcal{L}^{+}$is very large (an optimal class), the solutions we obtain are classical.

Let it be mentioned that though our setting is in many senses the most natural, it is not unique, and some applications lead to a different problem setting with non-classical solutions and moving boundaries.

\subsection{Motivation and approaches}

A main mathematical motivation to study equation (1.1) stems from the current interest in understanding the theory of fully-nonlinear parabolic equations with non-divergence form, for which this is an example with quite simple quadratic structure that offers a number of challenges. It is known that the set of solutions of (1.1) and their behaviour depends strongly on the value of the parameter $\gamma$ : though the theory is well-known for $\gamma<0$, it is still poorly understood for $\gamma>0$. Consequently, we restrict our analysis to $\gamma>0$, and in particular to the "good" subrange $\gamma>N / 2$, since the whole range offers too many novelties for a single study. This is a consequence of the rich structure of the problem.

As a token of the results that are found, let us just mention that a continuous, nonnegative and bounded viscosity solution (defined in a rather standard sense, following the theory of Crandall, Evans and Lions, cf. [25, 26, $23,24]$ ) is not necessarily determined in a unique way by its continuous initial trace, as we will show. In that sense, we point out that even in the selected range we have been forced to focus on a class of solutions with noncontracting supports, which is natural in some applications; but it has been subsequently pointed out by interested researchers that other classes enter their studies, more below.

The choice of this particular equation as the object of study is also motivated by a number of applications to diffusive phenomena in physics and biology. Let us mention several applications with parameter $\gamma \geqslant 0$, and comment on the results and the genesis of the paper at the same time. As a first example, it has been proposed as a model for the evolution of some biological populations and studied by Ughi and other authors, cf. [47, 29, 12, 13, 14]. In this context $v \geqslant 0$ is a density or a concentration, and our work is based on theirs. Thus, for all continuous and bounded initial data the authors obtain a unique solution by means of the vanishing viscosity method. These 
solutions are weak solutions in a suitable definition. The important progress done in those papers does not solve however the problem of well-posedness of the Cauchy problem with general data that is our main concern.

The equation is also used (in an equivalent form) to describe the cooling of a fireball produced by a strong explosion of a local gas, [43] and the special case $\gamma=1$ is studied in $[44,38]$.

In another context, Barenblatt et al. [7] have recently proposed the same equation to describe groundwater flow through a water-absorbing fissurized porous rock, and then $v \geqslant 0$ is the scaled groundwater level. Natural classes of solutions with shrinking supports are found. Such solutions are not in our class, and the new model represents different physics through its moving interfaces or free boundaries. It will be left out of our present considerations, and we refer to [51] where progress is done in understanding that setting. Finally, Barenblatt [6] has used an integrated version of this equation to analyze the process of contour enhancement in image processing, see also [8]. Again, a contracting free boundary appears, and it is basic in describing the moving contours.

\subsection{Usual concepts of solution and why they do not work}

Since the equation is only degenerate parabolic at the value $v=0$, a theory of classical solutions with bounded initial data such that $v_{0}(x) \geqslant \varepsilon>0$ is immediate, the problem is well-posed, the solutions are $C^{\infty}$ smooth, the Maximum Principle applies, and these facts hold independently of the value of $\gamma$.

However, such a nice panorama breaks down if the value $v=0$ is admitted. Among the problems that arise, the lack of uniqueness of different types of solutions is of major importance. We refer to Section 2 where an example of non-uniqueness of $C^{2}$ solutions with continuous initial data is given. There are several ways of defining classes of generalized solutions that are used for this type of equation. We list the usual options below and briefly explain why they do not provide for a suitable answer to the uniqueness question.

Classical Solutions. Equation (1.1) can be written in the equivalent form

$$
v_{t}=\frac{1}{2} \Delta\left(v^{2}\right)-(\gamma+1)|\nabla v|^{2} .
$$

In view of our existence results, we define a classical solution as a continuous function $v$ such that $\Delta\left(v^{2}\right)$ and $v_{t}$ are also continuous and the equation is satisfied everywhere. Note that such solutions need not be twice continuously differentiable in space. However, we will see that classical solutions are not uniquely defined by their initial data even if $u$ is a continuous function 
down to $t=0$. The non-uniqueness phenomenon has to do with the way the zeros of the initial data are taken, and how the zero-level set evolves in time. This will carefully explained in Subsection 1.3.

Vanishing Viscosity Method and Weak Solutions. As was said, the vanishing viscosity method (v.v.m.) was used by Ughi et al. to select the "good" solution. Given a bounded and continuous initial data $v_{0}$, it consists in adding a well-known viscosity term, $\varepsilon \Delta v$, which avoids degeneracy of the equation. Then, passage to the limit as $\varepsilon \rightarrow 0$ yields a weak solution which plays an important role, since it is maximal among the many possible weak solutions which take such initial data in the usual sense. Weak solutions are defined in a rather standard way, cf. Section 3. Thus, the authors of [29] study the related equation $u_{t}=u \Delta u+g(u)$ with zero Dirichlet boundary conditions, and show that nonnegative weak solutions exist, that they are not unique in general, and that exactly one maximal solution exists. They add that for "nice" initial data a maximal solution is characterized by the property that the support remains constant in time. As we show below, such characterization does not hold for more general data, even for continuous and bounded data. Hence, the problem of uniquely identifying the solutions remains partially unanswered. This is an important problem that we want to address here, since it is expected that when weak solutions can be naturally defined they provide for a class of uniqueness, and this happens for $\gamma<0$, but it is not true for $\gamma>0$.

Viscosity Solutions. Viscosity solutions (in the Crandall-Lions sense, see [23]-[26]) are used to select the "good" solution when uniqueness problems occur due to the lack of regularity. In the case $\gamma<0$, well-posedness of the pressure equation (1.1) has been established by Caffarelli and one of the authors [18] in the class of continuous and bounded solutions, using a modified concept of viscosity solution. However, a similar modification of this well-known framework does not work for $\gamma>0$ : it may help in choosing the setting of solutions with constant support, but it does not identify the correct concept of initial data that produces uniqueness.

\subsection{Outline of techniques and results}

Though the interested reader may proceed directly with the theory developed in Sections 2 to 8, we will spend some effort in presenting here the key issues, main steps and results of the paper.

The uniqueness theory developed below needs either the class $\mathcal{W}_{s}$ of weak solutions that have constant support in time with only quadratic zeros, cf. Section 3, or the class $\mathcal{C}_{q}$ defined as the subset of classical solutions with quadratic zeros. 
These are defined as follows: at every point $x_{0}$ and time $t_{0}>0$ where $u\left(x_{0}, t_{0}\right)=0$, we have $u\left(x, t_{0}\right)=O\left(\left|x-x_{0}\right|^{2}\right)$ for $x$ near $x_{0} . \mathcal{C}_{q}$ solutions are also shown to have constant support in time, and the two classes turn out to be identical in our problem setting. Let us point out from the start that there are solutions in those classes that cannot be obtained by the vanishing viscosity method, there are even such solutions with bounded and continuous data. Generally, we can treat initial data with arbitrary growth in the class $\mathcal{L}^{+}$.

The Transformation $T$. It is well-known that equation (1.1) can be formally mapped for $\gamma \neq 0$ into the well-known equation

$$
u_{t}=\Delta\left(u^{m}\right) \text {, }
$$

by means of the transformation $v=m u^{m-1}$, or equivalently,

$$
u=(v / m)^{-\gamma}, \quad \text { with } \quad m=(\gamma-1) / \gamma, \gamma=1 /(1-m) .
$$

We will write $v=T(u), u=T^{-1}(v)$ with definitions a.e. for weak solutions, everywhere for classical solutions. Though both equations are closely related, the mathematical investigation has focused mainly on (1.3) which, though degenerate parabolic, has a divergence structure and is well posed in several classes of weak solutions. There is a large literature on the issue, cf. $[3,50]$, mainly in the case $\gamma<0$, which corresponds to $m>1$. Then Equation (1.3) is the well-known porous medium equation. In this range $v$, solution of (1.1), is usual called the pressure, and $u$, defined by (1.4) and solution of (1.3), is the density, see [3]. The pressure formulation is most useful in describing dynamical properties, like moving interfaces. We will keep such terminology in this paper.

The extended theory of the FAst-Diffusion Equation. The results are quite different in the fast diffusion range $m<1$, i.e., for $\gamma>$ 0 , mainly due to the large number of solutions that can be constructed, cf. $[12,13,14]$, see also $[2,30,48]$ for related work (in these works, transformation $T$ is already used). Therefore, the main problem is uniqueness, more precisely, selecting the good solutions and characterizing them in terms of the data. We will perform below a detailed study of the range $\gamma>N / 2$ using as a key ingredient transformation (1.4). It still applies in the present situation, but in this range one must be careful in understanding the meaning of the equivalence of the $u$-and $v$-formulations, since $u \rightarrow 0$ implies $v \rightarrow \infty$ and $v \rightarrow 0$ implies $u \rightarrow \infty$. In particular, the degeneracy of equation (1.1) at the level $v=0$, which is the cause for the non-uniqueness problems pointed out by Bertsch et al., corresponds after the transformation to problems about the admissible singularities of $u$. 
In this direction, a general theory of existence and uniqueness for equation (1.3) has been only recently developed by the authors and allows $u$ to have permanent singularities in $Q$, even to be infinite on large measurable sets, [21]. We prove there that the Cauchy problem for (1.3) is well-posed in a class $\mathcal{E}_{c}$ of Extended Continuous Solutions (E.C.S.), taking as initial data any nonnegative Borel measure, $v_{0} \in \mathcal{B}^{+}$, an optimal class. The theory works perfectly on the condition that $m$ is restricted to the range

$$
m_{c}<m<1 \text {, with } m_{c}=(N-2)_{+} / N \text {, }
$$

which corresponds to $\gamma>N / 2$. We recall that Borel measures are the extension of nonnegative Radon measures to include possibly infinite values on certain Borel sets, cf. $[32,42]$ and Section 4 below. The class $\mathcal{E}_{c}$ consists of solutions which are continuous with values in $\mathbb{R}_{+} \cup\{+\infty\}$, having moreover only strong singularities (i.e., $u$ is not integrable in space in any neighborhood of any such singularity). The exact definitions and results of [21] will be recalled below for the reader's convenience.

Well-Posedness of the Pressure Equation in $\mathcal{W}_{s}$. Thanks to this extended theory and a careful analysis of the equivalence between both equations, we are able to show that the Cauchy Problem for equation (1.1) is wellposed in a suitable functional class for general initial data in the class $\mathcal{L}^{+}$of measurable nonnegative functions. The solution class is the transform of $\mathcal{E}_{c}$ via $T$. In this setting, the problem of uniqueness for the pressure equation is explained after introducing the concept of initial $p$-trace for a negative value of $p$, which corresponds to the usual Borel trace for the fast-diffusion equation. This is our key to uniqueness. Negative traces are a quite unexpected uniqueness tool in the parabolic literature, where the standard tools are the continuous trace or the $L^{1}$ trace. In other words, in the present situation the initial data are not "seen" in the usual way. Since this should also be the case for a number of other fully nonlinear equations, what is at the stake is the understanding of a new and effective notion of initial data.

A precise definition and study of the $p$-trace is done in Sections 5,6 . We restrict our analysis to the range $\gamma>N / 2$ and address in this general framework the questions of existence, uniqueness and related qualitative and quantitative questions which receive clear (and in some sense optimal) answers.

The reader may wonder why this limitation on the range. Actually, a number of features change for $\gamma \leqslant N / 2$, the theory is more complex, and actually it is still not well understood. In dimension $N=1$ we make the stronger restriction $\gamma>1$ so that still $m>0$. This is made to avoid the non-uniqueness problems associated with the range $-1<m \leqslant 0$, i.e. $\gamma \in(1 / 2,1]$. These problems are discussed in $[30,46]$ for instance. It must be remarked that most of the results of this paper are still true for this range, though we will not discuss it to avoid complicating the presentation with a side issue. 
Let us also mention that $p$-traces for positive values of $p$ may seem more "natural", but clearly such traces will not help in the present situation. Indeed, they do not allow for a precise description of the zero-level sets with local sinks, which is at the heart of non-uniqueness and we analyze next. We define a local sink as a spatially isolated zero of the solution, see the corresponding subsection in the Conclusions. Important sinks are those that are permanent in time.

Different Zeros, Non-uniqueness Phenomenon. The class $\mathcal{W}_{s}$ that gives uniqueness consists of weak solutions that have constant support in time. In other words, such solutions have constant zero-level set $\mathcal{Z}_{0}(v(t))=$ $\left\{x \in \mathbb{R}^{N}: v(x, t)=0\right\}$ for all $t>0$. Now, the $p$-trace for $v$ is precisely defined to correspond to the usual Borel trace for $u$. This leads us to distinguish two types of zeros for the initial data. The argument goes as follows: according to the theory of fast diffusion done in [21], a distinction must be made between weak and strong initial singularities of $u_{0}$. Weak singularities include integrable singularities and also locally bounded measures, like Dirac deltas. They are transformed by $T$ into so-called weak zeros of $v_{0}$. On the other hand, strong singularities of $u_{0}$ (which are not integrable) go into what we call intrinsic zeros of $v_{0}$.

The zero-level set of the initial data $v_{0}$ (even continuous initial data) contains information that may not be sufficient to provide uniqueness, since this property depends on the $p$-trace. What does it mean in practice? it has already been pointed out that while the intrinsic zeros remain as such for positive times, weak zeros may or may not disappear. Moreover, we show that one can decide to make a weak zero become intrinsic by changing $u_{0}$ (this is done by adding an "infinite Dirac mass"), which does not affect the initial data $v_{0}$. Hence the problem of non-uniqueness, since we obtain several solutions with the same initial data $v_{0}$. In other words, there are initial sinks which may disappear for $t>0$, and non-uniqueness is tied in the simplest case to the way they do it. A particularly simple example is explained in Section 2, where the data for $u$ are $u_{0, c}(x)=|x|^{-\alpha}+c \delta_{0}, c>0$, and the initial traces (in the standard continuous or $L_{l o c}^{p}$ sense, $1 \leqslant p \leqslant \infty$ ) of all $v_{c}$ 's are the same.

REsults. Performing the outlined process we get the following results:

(i) The setting of initial $p$-traces with $p=-\gamma$, data in $\mathcal{L}^{+}$, and solutions in $\mathcal{W}_{s}$ produces a well-posed Cauchy problem for Equation (1.1).

(ii) Our weak solutions (in $\mathcal{W}_{s}$ ) are in fact classical (which does not mean $C^{2}$ though), have constant support for all $t>0$, and possess only quadratic zeros, hence $\mathcal{C}_{q}=\mathcal{W}_{s}$.

These results are the main purpose of the paper. They are stated in full detail in Theorems 4.6, 5.1 and 5.4. 
(iii) We give the exact characterization of the solutions obtained by the vanishing viscosity method, see Theorem 5.5. We discuss the concepts of maximal and minimal solution associated to an initial measurable function $v_{0} \geqslant 0$.

(iv) We also show that singular measures are not allowed as initial data for $v$ in the following sense: let $\mu$ be a Radon measure in $\mathbb{R}^{N}$ which we decompose as

$$
d \mu=f(x) d x+d \mu_{s}
$$

where $f \in L_{\text {loc }}^{1}\left(\mathbb{R}^{N}\right)$ and $\mu_{s}$ is singular with respect to the Lebesgue measure. Then if we make a smooth approximation $\mu_{n}$ of $\mu_{s}$ and solve the problem with the data $f+\mu_{n}$, the associated solutions will converge to the unique solution in $\mathcal{W}_{s}$ which has initial data $f$. Thus, the singular part disappears in the limit. Let us remark that though there are no solutions with a Dirac mass as initial data, there are solutions with an inverse power of any singular measure as initial trace, like " $\delta_{0}^{-1 / \gamma}$ " (properly interpreted).

(v) Conversely, the pressure equation sheds light on the theory of Extended Continuous Solutions for (1.3). Indeed, though the theory of extended continuous solutions of [21] is based on passage to the limit of solutions which satisfy equation (1.3) in the whole space, either in the classical or the weak sense, the definition of extended solutions allows for no verification of the equation at the very hot set where $u=\infty$, and this set can be quite large. The re-formulation into (1.1) allows us to give sense to some equation at the infinite level set of $u$, since $v=0$, which allows to use a a distribution formulation, and even in the classical sense. In this respect, we can see the pressure change of variables as a type of renormalization of our extended theory of fast diffusion for $u_{t}=\Delta u^{m}$; however, the solutions are not renormalized solutions in the technical sense used in the recent literature on elliptic and parabolic equations, cf. [28, 15].

Distribution. The contents of the paper is distributed into three main parts as follows: in Section 2 we examine the concept of classical solution and suitable variants, give relevant examples and derive the property of support invariance, which is a main feature of our class of solutions. Section 3 deals with the concept of weak solution, as well as the vanishing viscosity approximation. We exhibit an example of classical solution that is not acceptable though it has constant support, but is has a nonquadratic zero. This ends the preliminaries.

We next address the question of well-posedness with optimal classes of initial data. We begin by examining in Section 4 the equivalence of the $v$-and $u$-formulations and deriving existence results from the theory constructed in [21]. 
In Section 5 we establish the existence and uniqueness of a classical solution for any given $p$-trace. We devote much effort to understand the initial trace in more standard form. In particular, we discuss the family of solutions associated to one initial function $v_{0} \in \mathcal{L}^{+}$and introduce the subclass $\mathcal{L}_{s}^{+}$. The class of solutions obtained as limits of the vanishing viscosity method is identified as the class of maximal solutions. It follows that not all solutions can be obtained by the vanishing viscosity method, though all of them are limits of $C^{\infty}$ and positive solutions obtained by suitably approximating the data.

Section 6 shows that the non-uniqueness problem related to the $p$-measures occurs even for continuous data. Section 7 deals with nonexistence for measures. In Section 8 there is a discussion of $L^{1}$ traces.

The last part of the paper concerns additional information. Thus, Section 9 studies the classes of separable, stationary and self-similar solutions and discusses the asymptotic behaviour of the solutions as $t \rightarrow+\infty$. Section 10 briefly discusses a Cauchy-Dirichlet problem. We devote another section to comment on the classes of solutions with contracting and expanding supports, which form a separate theory to be developed independently.

We end the article by a brief section on conclusions and comments. In particular, we make a comment about fixed and prescribable sinks, of interest for the application to diffusion.

Notations. (See exact definitions in next sections)

$C^{k}, C^{k, \alpha}(k \geqslant 0, \alpha \in(0,1))$ are the usual spaces of $k$-continuously (or $\alpha$-Hölderian) differentiable functions, $C_{0}$ is the space of continuous functions with compact support.

$\mathcal{L}^{+}$is the set of nonnegative measurable functions in $\mathbb{R}^{N}, \mathcal{L}_{s}^{+}$stands for weakly lower semi-continuous at zero functions in $\mathcal{L}^{+}$.

$\mathcal{W}$ is the class of weak solutions, cf. definition $(3.5) ; \mathcal{W}_{s}$ stands for weak solutions having constant zero-level set with only quadratic zeros.

$\mathcal{C}$ is the class of classical solutions, cf. definition in Section 2 next; $\mathcal{C}_{q}$ stands for classical solutions having only quadratic zeros.

$\mathcal{Z}_{0}(\cdot)$ is the zero-level set of a function, $\mathcal{Z}_{i}(\cdot)$ the set of intrinsic zeros, $\mathcal{Z}_{w}(\cdot)$ the set of weak zeros. See Sections 4,5 . 


\section{Classical solutions and examples}

We have said that there are a number of different concepts of solution that may be useful in the study of equation (1.1). Firstly, there is the concept of classical solution, by which we mean a nonnegative function $v \in C^{0}\left(Q_{T}\right)$, $Q=\mathbb{R}^{N} \times(0, T)$ for some $T>0(T=\infty$ allowed $)$, such that $\Delta\left(v^{2}\right)$ and $v_{t}$ are also continuous and the equation

$$
v_{t}=\frac{1}{2} \Delta\left(v^{2}\right)-(\gamma+1)|\nabla v|^{2}
$$

is satisfied everywhere in $Q_{T}$. Note that for $C^{2}$ solutions this equation is equivalent to (1.1), but it will be convenient to use this form in the sequel because it is the regularity that our solutions possess. Classical solutions form the class $\mathcal{C}$. A classical solution such that $\Delta v$ is continuous will be called strictly classical to make the difference clear. By a classical solution of the Cauchy problem for equation (1.1) with continuous and nonnegative data

$$
v(x, 0)=v_{0}(x), \quad x \in \mathbb{R}^{N},
$$

we understand a classical solution which is continuous in $Q=\mathbb{R}^{N} \times[0, T)$ and takes the value $v_{0}(x)$ at $t=0$. As we have already pointed out, standard quasilinear parabolic theory [41,33] applies to the class of classical solutions with strictly positive data, so a classical solution is $C^{\infty}$ smooth on the positivity set $\mathcal{P}(v)=\{v>0\}$ and the Strong Maximum Principle applies.

Explicit examples of Classical Solutions. There are a number of explicit classical solutions, like the constants $v=c, c \geqslant 0$. For $\gamma>N / 2$ a quite interesting example is provided by the quadratic solution

$$
V_{0}(x, t)=k \frac{|x|^{2}}{t}, \quad k=\frac{1}{2(2 \gamma-N)} .
$$

This classical solution takes on infinite initial data, $V_{0}(x, 0)=+\infty$, for every $x \neq 0$ in the (extended) continuous sense, while $V_{\infty}(0, t)=0$ for any $t>0$. In terms of the FDE (1.3) it is the transform of the solution with a strong singularity at $x=0$, so-called IPSS in [21]. The singularity takes now the form of a quadratic zero, one of the features of the theory of classical solutions that we will develop. The quadratic solution is strictly classical, but it admits a variant that is only classical and is nevertheless an acceptable solution in our theory, a limit of smooth and positive solutions:

$$
V_{+}(x, t)=k_{1} \frac{\left|x_{1}\right|^{2}}{t} \quad \text { for } x_{1} \geqslant 0, \quad V_{+}\left(x_{1}, t\right)=0 \quad \text { for } x_{1}<0,
$$


where $x=\left(x_{1}, \ldots, x_{N}\right)$ and $k_{1}=1 /(2(2 \gamma-1))$. By rotation of the axes we can define a number of variants, all of which vanish in a half-space for all $t>0$ and are not $\mathcal{C}^{2}$.

A related family of interesting smooth solutions with unbounded initial data are the transforms of the Barenblatt source-type solutions, which now read

$$
V_{A}(x, t)=\frac{k|x|^{2}+A t^{2 \theta / N}}{t}, \quad \theta=\frac{N \gamma}{2 \gamma-N},
$$

and $A$ is a positive constant which can be determined from the $L^{1}$-norm of the function $u(x, t)$ given in terms of $V$ by formula (1.4). The source-type solutions are positive and classical solutions, $C^{\infty}$ smooth. We observe next that when $A$ is taken as negative we also get a family of solutions

$$
V_{A}(x, t)=\frac{\left(k|x|^{2}-B t^{2 \theta / N}\right)_{+}}{t},
$$

with $\theta$ and $k$ as before, that we call for brevity pseudo-Barenblatt in [21]. They are supported in the contracting set $\{(x, t):|x| \geqslant r(t), r(t)=$ $\left.A^{1 / 2} t^{\theta / N}\right\}$. They are continuous but not $C^{1}$ and they have an interface or free boundary which separates the sets $\{v>0\}$ and $\{v=0\}$ and is given by the formula $|x|=r(t)$. The gradient jumps at the points of the interface, much as it happens in the theory of the porous medium equation $(\gamma<0)$. A related example is studied by Barenblatt et al. in [7], see Section 11 for details. These solutions are examples of weak solutions, a concept that we will define and discuss below, but they are not classical solutions and they do not fit into our present study.

Before we proceed, let us mention another typical family of non-classical solutions, the traveling waves with speed $c$ which have the form

$$
V_{c}(x, t)=\frac{c}{\gamma}\left(x_{1}-c t\right)_{+} .
$$

Then $V_{c}$ behaves linearly near the interface $\left\{x_{1}=c t\right\}$ as in the previous example. There is a large literature on the construction of self-similar solutions for this and similar nonlinear parabolic equations, cf. the previous references and [39, 40, 45].

A Counter-Example to the Uniqueness of Smooth Solutions. Let us consider initial data for the fast-diffusion equation of the form

$$
u_{0, c}(x)=|x|^{-\alpha}+c \delta_{0},
$$

where $\alpha<N$ and $c>0$. According to the theory of fast diffusion, cf. [21], and the transformation $T$ introduced above, any $c>0$ gives rise to a solution of the pressure equation $v_{c}$ by transformation of the solution $u_{c}$ of the 
associated fast-diffusion problem. All the $v_{c}$ 's are different, but they take on the same initial data $v_{0}(x)=m|x|^{\alpha / \gamma}$ continuously. Let us check that they are smooth: every $u_{c}$ is bounded below by the solution with $c=0$, which is positive, hence the $v_{c}$ 's are uniformly bounded from above. On the other hand, we know that every $u_{c}$ is locally bounded since the restriction $\alpha<N$ implies that it does not have strong singularities; it follows that $v_{c}$ is bounded below away from zero. In the absence of degeneracy, they are $C^{\infty}$ smooth for $t>0$. By a similar reason, they take the initial data in a $C^{\infty}$ way for $x \neq 0$, and only in a Hölder continuous way for $x=0$, since $u_{c}$ lies above the solution with data $|x|^{-\alpha}$ which is known to be self-similar of the form

$$
u(x, t)=t^{-r} f\left(x t^{-s}\right)
$$

for suitable $r$ and $s$ depending on $\alpha$, hence we have a bound above for $v_{c}$ at $(0,0)$. Note that the constructed solution is $C^{\infty}$ smooth for $t>0$. This example is essentially taken from [14].

We will prove below that when the initial data are regular enough the classical solution is unique, Theorem 6.3.

Support Properties. The qualitative difference just observed between the examples of classical solutions and the other examples is reflected in the following general principle: classical solutions have non-contracting supports. To prove that result we need a preliminary Lemma.

Lemma 2.1 There is a positive and smooth function $w(x, t)$ defined in the contracting domain

$$
K=\left\{(x, t):|x-a|^{2}<b-c t\right\}
$$

for given $a, b, c>0$ with the following properties: it is a classical subsolution of equation (1.1) in the set $w>0$, it is $C^{1}$ up to the lateral boundary, where it takes zero value and nonzero space derivative. The $L^{\infty}$ norm of $w$ can be chosen as small as we please.

Proof. By classical subsolution we mean that equation (1.3) is replaced by the inequality

$$
v_{t} \leqslant \frac{1}{2} \Delta\left(v^{2}\right)-(\gamma+1)|\nabla v|^{2},
$$

valid for all points where $v>0$. Without loss of generality we can take $a=0$. Here is the construction

$$
w(x, t)=A\left(b-x^{2}-c t\right)_{+},
$$


with $A>0$ to be chosen. It is positive for $x^{2} \leqslant b-c t$. The calculation of the subsolution condition gives

$$
c \geqslant 2 A b N-2 A(N-2) x^{2}-2 A N c t .
$$

Hence a sufficient condition for $N \geqslant 2$ is $2 N b A \leqslant c$, for $N=1$ we have $4 A b \leqslant c$. This determines $A$ so that $\|w\|_{\infty}=A b$ is of the order of $c$ or less.

Here is the precise version of the result on the supports and level sets. It holds under a slightly less stringent requirement than that of classical solution.

Proposition 2.2 Let $v \geqslant 0$ be a $C^{1}$ function defined in a closed cylinder $Q=\Omega \times I, \Omega$ the closure of a bounded domain, $I=[0, T]$, that solves equation (1.1) in the set $\{(x, y) \in Q: v(x, t)>0\}$. Then the zero-level set does not expand in time.

Proof. More technically, the assertion of the Proposition means that if we put $\mathcal{Z}_{0}(t)=\{x \in \Omega: v(x, t)=0\}$, then the family $\mathcal{Z}_{0}(t)$ is nonincreasing in time. Alternatively, we can say that the positivity sets of the solutions do not contract.

For the proof, suppose that $x_{0}$ is a point in $\Omega$ such that $v\left(x_{0}, 0\right)>0$ and let us prove that $v(x, t)>0$ in a space neighborhood $x \in B_{r}\left(x_{0}\right)$ for all $t \in[0, T]$. We may assume that $r$ is so small that $v\left(x_{0}, 0\right) \geqslant \varepsilon>0$ in the ball of radius $2 r$.

As a consequence of the lemma, then we take $a=x_{0}, b=2 r$ and $c$ very small so that $a-T c \geqslant r$ and study the difference $v-w$ in the set $K \subset Q$. It is clear that $v(x, 0)-w(x, 0)$ is positive for $t=0$. By standard regularity theory $v$ is $C^{\infty}$ smooth where it is positive. If $v-w$ is not positive in $K$ there is a first time $t_{1}$ where the difference vanishes, let us call the point $y$. It cannot be an interior point because of the strong maximum principle for smooth solutions of fast diffusion. But, it cannot lie at the boundary, because then $v=w=0, \nabla v=0$ because $v$ is $C^{1}$ and we have a radial derivative $\nabla w \cdot \nu \neq 0$ (with $\nu$ the spatial outer normal at a point of the boundary of $K$ ). This implies that $v-w$ had to be negative somewhere before. Therefore, we conclude that $v \geqslant w>0$ in the interior of $K$, and since the interior of $K$ contains the line $\left\{\left(x_{0}, t\right): 0 \leqslant t \leqslant T\right\}$, the result is proved.

The next question that comes up is: can the solutions have really contracting zero-level set? The answer is that classical solutions can, see Section 11 , but the class of classical solutions with which we deal will not for $t>0$. Indeed, let us introduce the following definition 
Quadratic zeros. A zero of $v$ at the point $\left(x_{0}, t_{0}\right) \in Q$ is said to be quadratic (in space) if $u\left(x, t_{0}\right)=O\left(\left|x-x_{0}\right|^{2}\right)$ near $x=x_{0}$.

Since strict classical solutions have continuous $\Delta v$, it happens that all their zeros are quadratic, but the assertion for classical solutions is not automatic. We denote the class of classical solutions with quadratic zeros by $\mathcal{C}_{q}$. It will play an important role in what follows. The explicit solutions $V_{+}, V_{-}$, which are not strictly classical solutions, are at least in $\mathcal{C}_{q}$. In this class we can prove the property of constancy of the support:

Proposition 2.3 Let $v \geqslant 0$ be a classical solution in the class $\mathcal{C}_{q}$. Then the zero-level set

$$
\mathcal{Z}_{0}(t)=\{x \in \Omega: v(x, t)=0\}
$$

is constant in time for $0<t<T$.

Proof. It is based on comparison with positive $C^{\infty}$ solutions using the transformation into the $u$-formulation, a scheme that will be used repeatedly in the paper. We only have to prove that the zero-level set does not contract. So, if $x_{0}$ is a zero of $v$ at time $t_{0}$ we know that it has a quadratic behaviour, i.e., $v\left(x_{0}, t_{0}\right) \leqslant C\left|x-x_{0}\right|^{2}$ for some $C>0$ in a neighborhood of $x_{0}$. Let us put $t_{0}=0$ without loss of generality (shift the origin of time), and let us approximate $v(\cdot, 0)$ from above by smooth and positive functions $v_{n}(\cdot, 0)$ that converge monotonically to $v(\cdot, 0)$. The solutions to the approximate problems exist, are unique and are ordered. The transforms $u_{n}(x, t)$ are a monotonically increasing family whose initial data approximate a function $u(x, 0) \geqslant C_{1}\left|x-x_{o}\right|^{-2 /(1-m)}$ which is not integrable. According to the theory of [21], it creates a standing strong singularity in the limit, so that

$$
\lim _{n \rightarrow \infty} u_{n}\left(x_{0}, t\right)=\infty
$$

for every $t>0$. In terms of $v$ it means that $v=\lim _{n \rightarrow \infty} v_{n}$ has a zero at $x=x_{0}$ for all $t \geqslant 0$. It also follows from [21] that the zero must be quadratic for $t>0$ if it was for $t=0$.

In view of the proof and of the exact condition for a standing strong singularity found in [21], we see that the condition of quadratic zero can be relaxed into the form of strong zero to be defined in Section 5 and used in the analysis of initial traces. However, both conditions turn out to be equivalent for $t>0$.

We will see further examples with separable and self-similar solutions in Section 9 . 


\section{Weak solutions. The vanishing viscosity approxima- tion}

In the paper [12], Bertsch and Ughi solve the initial-value problem for all $\gamma \geqslant 0$ with bounded, nonnegative and continuous initial data by the method of vanishing viscosity, i.e, they solve the approximate problem

$$
v_{\varepsilon, t}=v_{\varepsilon} \Delta v_{\varepsilon}-(\gamma+1)\left|\nabla v_{\varepsilon}\right|^{2}+\varepsilon \Delta v_{\varepsilon}, \quad v_{\varepsilon}(x, 0)=v_{0}(x), \quad \varepsilon>0,
$$

which turns out to be equivalent to

$$
\bar{v}_{\varepsilon, t}=\bar{v}_{\varepsilon} \Delta \bar{v}_{\varepsilon}-(\gamma+1)\left|\nabla \bar{v}_{\varepsilon}\right|^{2}, \quad \bar{v}_{\varepsilon}(x, 0)=v_{0}(x)+\varepsilon,
$$

with $\bar{v}_{e}=v_{\varepsilon}+\varepsilon$. By the classical theory the solutions $\bar{v}_{\varepsilon}$ exist, are unique and smooth and they form a monotone family as $\varepsilon \searrow 0$, which allows to define the limit solution

$$
\bar{v}(x, t)=\lim _{\varepsilon \rightarrow 0} \bar{v}_{\varepsilon}(x, t)=\lim _{\varepsilon \rightarrow 0} v_{\varepsilon}(x, t) .
$$

The authors call this solution the viscosity solution, but we will call it vanishing-viscosity solution and use the name viscosity with another meaning according to current usage. They are able to prove that the limit is a continuous function if $\gamma>N / 2$ (limit solutions can be discontinuous for $0 \leqslant \gamma<1, N \geqslant 2[13])$. They also show that the limit is a weak solution in the following sense: $v \in L^{\infty}\left(Q_{T}\right) \cap L^{2}\left(0, T ; H_{l o c}^{1}\left(\mathbb{R}^{N}\right)\right)$ and

$$
\int_{\mathbb{R}^{N}} v_{0}(x) \varphi(x, 0) d x+\iint_{Q}\left\{v \varphi_{t}+\left(v^{2} / 2\right) \Delta \varphi-(1+\gamma)|\nabla v|^{2} \varphi\right\} d x d t=0
$$

for every test function $\varphi \in C^{2,1}\left(\mathbb{R}^{N} \times[0, T)\right)$. The limit solution obtained from the vanishing viscosity method is maximal in the class of continuous weak solutions, but they show that there is no uniqueness of the continuous weak solution with a given initial data taken continuously.

We will be interested in considering general initial data. Hence, our definition of weak solution is adapted as follows. A continuous weak solution of equation (2.1) is a function $v \in C^{0}(Q), v \geqslant 0$, with $\nabla v \in L_{l o c}^{2}(Q)$ such that

$$
\iint_{Q_{T}}\left\{v \varphi_{t}+\left(v^{2} / 2\right) \Delta \varphi-(1+\gamma)|\nabla v|^{2} \varphi\right\} d x d t=0
$$

for every compactly supported test function $\varphi \in C^{2,1}\left(Q_{T}\right)$. We denote by $\mathcal{W}$ the class of such solutions. This class is convenient as a reference framework but it is too large, it is not a class of uniqueness. 
It is easily checked that classical solutions are weak solutions, and so are the pseudo-Barenblatt and the traveling waves. A further example of interest is provided by the stationary solution

$$
v(x, t)=C|x|^{\sigma}, \quad \sigma=\frac{N-2}{\gamma-1}
$$

$(C>0)$, valid for $N \geqslant 3$. This solution has a zero at $x=0$ and is a classical solution in our sense if $\sigma>1$, i.e., when $\gamma<N-1$ or $m<(N-2) /(N-1)$. But since $\sigma$ is always less than 2 in our range of $\gamma$, the zero is never quadratic and it is never a strict classical solution. For $N=1,2$ see Section 9. The stationary solution will not be an acceptable solution in our existence and uniqueness theory. In particular, it is not a limit of a vanishing viscosity method or similar approximation procedure. Technically, we eliminate it because it does not have a quadratic zero.

There is still another option to study our equation, the modern concept of viscosity solution in the sense of Crandall and Lions. We delay the discussion of this concept in order to present our results (see last Section).

\section{Existence with optimal data through the transfor- mation to fast diffusion. The class of special solu- tions $\mathcal{W}_{s}$}

Transformation (1.4) allows us to enlarge the class of initial data considered so far and deal with any measurable function as initial data by translating the results of [21]. Let us recall the main results concerning the extended theory for equation (1.3), contained in that paper. We recall that the theory applies for $m_{c}<m<1$, i.e., for $\gamma>N / 2$, an assumption we will make in the sequel unless explicit mention to the contrary. Firstly, we need to introduce the proper class of solutions in fast diffusion, called extended continuous solutions:

The class $\mathcal{E}_{c} . \quad$ A function $u: Q \mapsto \mathbb{R}_{+} \cup\{+\infty\}$ belongs to the class $\mathcal{E}_{c}$ if (i) It is continuous as an extended function: $u \in \mathcal{C}^{0}\left(Q, \mathbb{R}_{+} \cup\{+\infty\}\right)$,

(ii) It is a distributional solution of (1.3) in the regular set $\Omega=\{(x, t) \mid$ $u(x, t)<\infty\} \subset Q_{T}$.

(iii) For every $t>0$ the singular set $\mathcal{S}(t)=\{x: u(x, t)=\infty\}$ is constant in time and consists of strong singularities, which means that for any $t, r>0$, and $y \in \mathcal{S}(t)=\mathcal{S}$,

$$
\int_{B_{r}(y)} u(x, t) d x=+\infty .
$$


A solution $u \in \mathcal{E}_{c}$ of the Cauchy Problem with initial data a Borel measure $\nu \geqslant 0$ is a function satisfying in addition:

(iv) The solution $u(\cdot, t)$ takes on the initial value $\nu$ in the sense of Borel trace as $t \rightarrow 0$, i.e., for any $\varphi \in C_{0}\left(\mathbb{R}^{N}\right)$ (i.e. continuous, compactly supported), $\varphi \geqslant 0$,

$$
\int_{\mathbb{R}^{N}} u(x, t) \varphi(x) d x \underset{t \rightarrow 0}{\longrightarrow} \int_{\mathbb{R}^{N}} \varphi(x) d \nu(x) \in \mathbb{R}_{+} \cup\{+\infty\} .
$$

Let us recall that any Borel measure $\nu \geqslant 0$ may be described as a couple $(\mathcal{S}, \mu)$, where $\mathcal{S}$ is the singular set, where $\nu$ takes on infinite value (it blows up), and $\mu \geqslant 0$ is a (locally finite) Radon measure on $\mathcal{R}=\mathbb{R}^{N} \backslash \mathcal{S}$. The following results are proved in [21]:

Theorem 4.1 Let $m_{c}<m<1$. Then for any Borel measure $\nu \geqslant 0$ in $\mathbb{R}^{N}$, there exists a unique solution $u \in \mathcal{E}_{c}$ such that $u(0)=\nu$ in the sense of Borel trace. Moreover, $u>0$ in $Q_{\mathcal{R}}=\mathcal{R} \times(0, \infty), u \in C^{\infty}\left(Q_{\mathcal{R}}\right)$, and $u_{t}=\Delta u^{m}$ in the classical sense in $Q_{\mathcal{R}}$. The Maximum Principle applies and the solutions depend continuously on the data in the natural topologies for the spaces of data and solutions.

We also have the following estimate of the behaviour near a singularity, that we called the Radiation Lemma in [21].

Proposition 4.2 If $\mathcal{S} \neq \emptyset$, then for every $x \in \mathbb{R}^{N}, t>0$

$$
u(x, t) \geqslant\left(\frac{C t}{\operatorname{dist}(x ; \partial \mathcal{S})^{2}}\right)^{\frac{1}{1-m}}, \quad C=\frac{2 m}{1-m}(2-N(1-m)) .
$$

This estimate has a clear consequence in terms of the pressure; $v=$ $m u^{m-1}$ is uniformly continuous near a point where $v=0$ if $t \geqslant \tau>0$. Further quantitative estimates are the following.

Proposition 4.3 For every E.C.S. in the range $m_{c}<m<1$ we have the following estimates: $(i) u_{t} / u$ is bounded for any $t>0$. More precisely,

$$
-\frac{\theta u}{t} \leqslant u_{t} \leqslant \frac{u}{(1-m) t}, \quad \theta=(m-1+2 / N)^{-1} .
$$

(ii) There are also bounds on spatial derivatives of $v=m u^{m-1}$ :

$$
-\frac{1}{t} \leqslant \Delta v \leqslant \frac{C_{1}}{t}, \quad C_{1}=\theta(1-m),
$$

and

$$
|\nabla v|^{2} \leqslant C_{2} \frac{u^{m-1}}{t}, \quad C_{2}=2 \theta(1-m) / N .
$$

(iii) Moreover, when the initial trace is $(\mathcal{S}, 0)$, we have $u_{t}>0$ in $Q_{\mathcal{R}}$. 
We now try to apply backwards the transformation (1.4) to the solution of the fast diffusion equation (1.3). This is not so easy when dealing with such a large class of solutions. The transformed function $v$ is a continuous function because of the uniform behaviour of $u$ near the singular set. Moreover, $v$ solves equation (1.1) in the classical sense on the set $\{v>0\}$. Let us examine the situation at $v=0$ more closely: the zero-level set of $v$ is constant in time (and coincides with the strongly singular set of the initial Borel trace of $u$ ). Let us call it $\mathcal{Z}_{0}$. The behaviour of $v$ around a point $x_{0} \in \mathcal{Z}_{0}$ is given by the last property of Theorem 4.1:

$$
v(x, t) \leqslant k \frac{\left(x-x_{0}\right)^{2}}{t}, \quad k=(2(2 \gamma-N))^{-1} .
$$

Because of (4.6) we recall the notion of quadratic zero as defined at the end of Section 2 and introduce the suitable class solutions

The special class $\mathcal{W}_{s}: A$ weak solution $v \in \mathcal{W}$ belongs to the subclass $\mathcal{W}_{s}$ if it has a constant zero-level set $\mathcal{Z}_{0}(v)=\left\{x \in \mathbb{R}^{N}: v(x, t)=0\right\}$ for $0<t<T$, and $\mathcal{Z}_{0}(v)$ consists only of quadratic zeros.

We remark that a similar pair of restrictions allow to define the subclass of special classical solutions $\mathcal{C}_{q}$, but in this case the restriction to quadratic zeros implies constancy of the support.

We can now state and prove the main equivalence result.

Theorem 4.4 There is a one-to-one correspondence between solutions $v \in$ $\mathcal{W}_{s}$ of (1.1) and solutions $u \in \mathcal{E}_{c}$ of equation (1.3). Moreover, the solutions $v \in \mathcal{W}_{s}$ are $C^{1}$ in $(x, t)$ and they are classical solutions for the equation written in the form

$$
v_{t}=\Delta\left(v^{2} / 2\right)-(\gamma+1)|\nabla v|^{2} .
$$

This means that the classes $\mathcal{W}_{s}$ and $\mathcal{C}_{q}$ coincide. Finally, $v$ enjoys the properties:

(i) for $t>0$, we have $v(x, t)=O\left(|x|^{2}\right)$ as $|x| \rightarrow \infty$, and when $\mathcal{S} \neq \emptyset$ then

$$
v(x, t) \leqslant \kappa \frac{\operatorname{dist}(x, \mathcal{S})^{2}}{t}, \quad \kappa=\frac{1}{2(2 \gamma-N)} .
$$

(ii) We also have the estimates

$$
|\nabla v|^{2} \leqslant C_{1} \frac{v}{t}, \quad-\frac{v}{t} \leqslant v_{t} \leqslant C_{2} \frac{v}{t},
$$

with $C_{1}=4 \kappa, \quad C_{2}=2 N \kappa$.

(iii) $\Delta v$ is bounded for any $t>0$. More precisely, we have

$$
-\frac{1}{t} \leqslant \Delta v \leqslant \frac{C_{2}}{t}
$$


Proof. Let $v=m u^{m-1}$ be the transform of an E.C.S. $u$. The quantitative estimates $(i),(i i)$ and $(i i i)$ are direct consequences of the results stated above for $\mathcal{E}_{c}$. If $u$ is a smooth solution (i.e., if $\mathcal{S}=\emptyset$ ) then $v$ is a classical solution with $\mathcal{Z}_{0}(v)=\emptyset$. More generally, we use the fact that any solution $u \in \mathcal{E}_{c}$ is the limit of smooth solutions $u_{n}$ with bounded data. Passing to the limit in the weak formulation for the corresponding $v_{n}$ and using the estimates and the discussion preceding the definitions of the special classes we conclude that $v$ belongs to $\mathcal{W}_{s}$.

For the continuity of the first derivatives near a point where $v(x, t)>0$, we use standard parabolic theory, $[33,41]$. Near a point where $v(x, t)=0$, $t>0$, the continuity of $v$ implies by virtue of the a priori estimates that both $\nabla v$ and $v_{t}$ tend to zero. This proves that actually $v \in \mathcal{C}_{q} \subset \mathcal{W}_{s}$. The reader is invited to check the details.

For the converse inclusion, any continuous weak solution is $C^{\infty}$ smooth where $v>0$ as a consequence of standard parabolic theory, hence the transform $u$ is a smooth solution of equation (1.1) in $\{(x, t): u(x, t)<\infty\}$. The special class $\mathcal{W}_{s}$ implies that the singular set is constant and the singularities are strong. Hence, $u \in \mathcal{E}_{c}$.

Remark. Though all the terms appearing in the equation under the form (2.1) are continuous functions in $Q, \Delta v$ need not be continuous as the example (2.4) and its variants show.

We list a consequence of the equivalence theorem

Corollary 4.5 The class $\mathcal{W}_{s}$ consists precisely the smooth positive solutions of equation (1.1) and its limits under locally uniform convergence.

However, not all the solutions of the initial-value problem can be obtained by the vanishing viscosity method as will see below, cf. Theorem 5.5. As yet another consequence, we get a preliminary existence result with general initial data.

Theorem 4.6 For any measurable function $v_{0}: \mathbb{R}^{N} \rightarrow \mathbb{R}_{+} \cup\{+\infty\}$, there exists at least a classical solution $v$ of equation (1.1) in $\mathcal{C}_{q}$ taking the initial data in the sense of almost everywhere convergence as $t \rightarrow 0$. The solution has the properties stated in Theorem 4.4.

The statement about convergence to the initial data follows from the Fatou theorem in [21]. In fact, if $v_{0}$ is more regular, the initial data are taken in a better sense. This will be discussed later, since we need first to address the questions of uniqueness and initial traces. 


\section{5. $p$-Traces and uniqueness}

It has been pointed out in previous works like [12] that uniqueness of weak solutions of the Cauchy problem does not hold, and this happens even if we assume smooth initial data and special continuous solutions as we will see in the next section. Thus, there is a problem in identifying the initial information that characterizes solutions in $\mathcal{W}_{s}$. The uniqueness question will be completely solved in the class $\mathcal{W}_{s}$ by means of the concept of Borel $p$-trace. We will also need to clarify the relation of the new concept with traditional concepts of trace. Note that the restriction to the class $\mathcal{W}_{s}$ is necessary, as counter-examples show (see Remarks below).

Definition. A (classical or weak) solution $v$ of equation (1.1) has a p-trace $\nu \in \mathcal{B}^{+}\left(\mathbb{R}^{N}\right)$ if $v^{p}(t)$ has the trace $\nu$ (in the usual Borel sense) as $t$ goes to zero, i.e., for any $\varphi \in C_{0}\left(\mathbb{R}^{N}\right), \varphi \geqslant 0$,

$$
\int_{\mathbb{R}^{N}} v^{p}(x, t) \varphi(x) d x \underset{t \rightarrow 0}{\longrightarrow} \int_{\mathbb{R}^{N}} \varphi(x) d \nu(x),
$$

whether the right-hand side is finite or not.

We can define a general notion of $p$-trace for all $p \in \mathbb{R}$, but the only case we are interested is the unusual negative value $p=-\gamma<-N / 2$, since our interest is in looking for the trace of the transform $u$ of a solution $v$, as we know from [21] that such traces exist in the sense of Borel measures if $u \in \mathcal{E}_{c}$.

Let us go for the details. We recall that every $\nu \in \mathcal{B}^{+}\left(\mathbb{R}^{N}\right)$ can be written uniquely as $\nu=(\mathcal{S}, \mu)$ with $d \mu=f(x) d x+d \mu_{s}, f \in L_{l o c}^{1}\left(\mathbb{R}^{N}\right)$ and $\mu_{s}$ a singular Radon measure. We also write $\nu=\left(\mathcal{S}, f, \mu_{s}\right)$. Let $v_{0}$ be defined a.e. in $\mathbb{R}^{N}$ by the rule $v_{0}(x)=m f(x)^{m-1}$. As a consequence of Theorem 4.1 and the Equivalence Theorem 4.4 we have

Theorem 5.1 For every nonnegative measure $\nu=(\mathcal{S}, \mu) \in \mathcal{B}^{+}\left(\mathbb{R}^{N}\right)$ there exists a unique solution of equation (1.1) in $\mathcal{W}_{s}$ which takes on the initial data $\nu$ in the sense of $p$-trace with $p=-\gamma$. The zero set of $v$ for all $t>0$, $\mathcal{Z}_{0}(v)$ coincides with $\mathcal{S}$. The solution is actually classical, $u \in \mathcal{C}_{q}$.

Conversely, any solution $v \in \mathcal{W}_{s}$ has an initial p-trace in the set $\mathcal{B}^{+}\left(\mathbb{R}^{N}\right)$ of nonnegative Borel measures. Dependence with respect to the initial data in the sense of p-traces holds.

Convergence of $v(x, t)$ to $v_{0}(x)$ as $t \rightarrow 0$ occurs for almost every $x$ along non-tangential cones. Convergence towards 0 holds everywhere in $\mathcal{S}$ along larger sets, of the form $\left\{\left|x-x_{0}\right|=o\left(t^{1 / 2}\right), t>0\right\}, x_{0} \in \mathcal{S}$. 
Proof. Given $\nu$ we know that there exists a unique E.C.S. $u$ taking the initial data $\nu$ in the sense of Borel trace. Defining $v=T(u)$ gives a solution of (1.1) which takes on the initial data in the sense of $\gamma$-trace. Moreover, any other solution $v^{\prime} \in \mathcal{W}_{s}$ which takes on the same initial data in the sense of $(-\gamma)$-trace will give rise to a $u^{\prime}$ with initial trace $\nu$. Thus $u^{\prime}=u$, and $v^{\prime}=v$ which proves uniqueness in $\mathcal{W}_{s}$. Finally, the approximation property comes from continuous dependence with respect to initial traces for (1.3) in $\mathcal{E}_{c}$ (in fact, this process is monotone). The convergence of $v(x, t) \rightarrow 0$ as $t \rightarrow 0$ for $x \in \mathcal{S}$ comes from the Radiation Lemma, the convergence in $\mathcal{R}=\mathbb{R}^{N} \backslash \mathcal{S}$ will be discussed next.

Therefore, a solution $v \in \mathcal{W}_{s}$ of (1.1) is uniquely determined by its $p$ trace, $p=-\gamma$. We devote the rest of the section to the important question of understanding how the initial information is related to $v_{0}$.

For data $v_{0}(x) \geqslant \varepsilon>0$ this is easy because then $u_{0}(x) \leqslant C \varepsilon^{-\gamma}$, there is a unique bounded solution $u$ and the data are taken in the $L_{l o c}^{p}$ sense for every $p<\infty$ and a.e. More generally, we have the following useful result of [21].

Proposition 5.2 Let $u \in \mathcal{E}_{c}$ and let us assume that the initial trace $\nu$ is given in a neighborhood $U$ of a point $x_{0} \in \mathbb{R}^{N}$ as a function $d \nu=f(x) d x$ which is continuous at $x_{0}$. Then

$$
\lim _{t \rightarrow 0, x \rightarrow x_{0}} u(x, t)=f\left(x_{0}\right) .
$$

If function $f$ is only locally integrable then the convergence is a.e. in the non-tangential sense.

In order to proceed further, we have to analyze the behaviour of solutions with data that are not strictly positive. Then it can happen that $u_{0}=c v_{0}^{-\gamma}$ is singular and we have to introduce the concept corresponding to a strong singularity (see (iii) in the definition of $\mathcal{E}_{c}$ ), which leads to the concept of strong zero for the initial data:

Intrinsic (or strong) zeros. A point $x_{0} \in \mathbb{R}^{N}$ is an intrinsic (strong) zero of a measurable function $v_{0} \geqslant 0$ if it is a strong singular point of $u_{0}=c v_{0}^{-\gamma}$, i.e. if

$$
\int_{U} v_{0}^{-\gamma}(x) d x=\infty
$$

in every neighborhood $U$ of $x_{0}$.

We use the standard convention $\infty \cdot 0=0$ to integrate infinite values of $u_{0}$ contained in a set of measure zero. The concept of intrinsic zero of $v_{0}$ is therefore dependent on the equation through the constant $\gamma$. It is invariant under changes of $v_{0}$ in a set of measure 0 . We define $\mathcal{Z}_{i}\left(v_{0}\right)$ as the set of intrinsic strong zeros of $v_{0}$. It is a closed subset of $\mathbb{R}^{N}$. 
We are now ready to discuss the extension of the transformation (1.4) to the class of measures as initial data, $\mathcal{B}^{+}$.

Definition. For every $\nu=\left(\mathcal{S}, f, \mu_{s}\right) \in \mathcal{B}^{+}\left(\mathbb{R}^{N}\right)$ we define the transform $v_{0}=T(\nu) \in \mathcal{L}^{+}\left(\mathbb{R}^{N}\right)$ by the formula

$$
\begin{cases}v_{0}(x)=m f(x)^{m-1} & \text { a.e. in } \mathcal{R}=\mathbb{R}^{N} \backslash \mathcal{S} \\ v_{0}(x)=0 & \text { in } \mathcal{S}\end{cases}
$$

Remarks. i) With this definition, $v_{0}$ is defined everywhere on $\mathcal{S}$ but only a.e. away from $\mathcal{S}$. Clearly, this is an extension of the transformation defined in (1.4) for functions and is compatible with taking limits. ii) The definition agrees with the expected monotonicity of $T$ that implies that $\mathcal{Z}_{i}\left(v_{0}\right)$ must be a subset of $\mathcal{S}$. In the class $\mathcal{E}_{c}$ this set is conserved in time and gives rise after the transformation $v=m u^{m-1}$ to the set of quadratic zeros of $v$. iii) If we assume that $f(x)$ is everywhere finite in $\mathcal{R}$, then $v_{0}$ is positive on $\mathcal{R}$. But we may prefer to put $v_{0}=0$ on the support of $\mu_{s}$.

Proposition 5.3 The image by $T$ of $\mathcal{B}^{+}\left(\mathbb{R}^{N}\right)$ is the set of nonnegative measurable extended functions $v_{0} \geqslant 0$ such that

$$
\mathcal{Z}_{i}\left(v_{0}\right) \subseteq\left\{x \in \mathbb{R}^{N}: v_{0}(x)=0\right\} \equiv \mathcal{Z}_{0}\left(v_{0}\right) .
$$

We call this property weak lower semicontinuity at 0, and the class of such functions $\mathcal{L}_{s}^{+}$. Conversely, for any such $v_{0}$, any closed set $\mathcal{S}$ satisfying

$$
\mathcal{Z}_{i}\left(v_{0}\right) \subset \mathcal{S}
$$

any singular Radon measure $\mu_{s}$ satisfying $\operatorname{supp}\left(\mu_{s}\right) \subset \mathbb{R}^{N} \backslash \mathcal{S}$, there is a unique $\nu=\left(\mathcal{S}, f, \mu_{s}\right)$ such that $v_{0}=T(\nu)$ up to a.e. equivalence in $\mathbb{R}^{N} \backslash \mathcal{S}$.

Proof. Most of it is a careful application of the definitions. In defining $T^{-1}$ we also need to observe that

$$
\operatorname{meas}\left(\mathcal{Z}_{0} \backslash \mathcal{Z}_{i}\right)=0
$$

in particular, $\operatorname{meas}\left(\mathcal{S} \backslash \mathcal{Z}_{i}\right)=0$. The proof is as follows: if the measure is positive, the set $\mathcal{Z}_{0} \backslash \mathcal{Z}_{i}$ has a point of density, but this point is so surrounded by zeros that it must be an intrinsic zero, hence a contradiction.

Remarks. iv) The set $\mathcal{S}$ is not determined uniquely by $v_{0}$. It will play the role of strong singular set for $\nu$, hence it can be labeled as the set of strong zeros. We need this additional information to $v_{0}$ as initial data for equation (1.1). 
v) A lower semi-continuous function at 0 will verify condition (5.2). In that case $\mathcal{Z}_{0}$ is a well-defined closed set.

vi) A minimal inverse to $T$ exists and consists in choosing $\mathcal{S}=\mathcal{Z}_{i}\left(v_{0}\right)$, and $\mu_{s}=0$. This gives rise to a minimal solution $u$ of (1.3) and consequently, to a maximal solution $v$ of (1.1).

vii) In general, there is no maximal inverse to $T$, since we can add any discrete number of points to $\mathcal{Z}_{i}\left(v_{0}\right)$ to form different sets $\mathcal{S}$ which are not ordered. A concept of maximal transform $T$ and minimal solution $v$ can be discussed for regular data, see Section 6 .

viii) About the freedom in the choice of $\mu_{s}$ let us point out that there exist nonnegative singular measures with arbitrary zero-measure closed support. For instance, if $K$ is a compact subset of $\mathbb{R}^{N}$ with positive $C_{\alpha, p^{-}}$capacity, then the capacitary measure $\mu_{K}$ of $K$ is a good example, and in fact, there are infinitely many. We refer to [1] for precise statements and related facts (see especially Thm 2.2.7. of this reference).

Now, let us examine the situation when $v_{0}$ is not weakly lower semicontinuous at zero:

Definition. For initial data $v_{0} \in \mathcal{L}^{+}$which are not weakly lower semicontinuous at zero, we re-define $v_{0}$ by putting $v_{0}=0$ on $\mathcal{Z}_{i}\left(v_{0}\right)$. This modification only affects $v_{0}$ on a set of zero Lebesgue measure in the zerolevel set and yields an initial data in $\mathcal{L}_{s}^{+}$that we still denote by $v_{0}$ when no confusion is to be feared.

We will explain below what happens if we do not re-define $v_{0}$ (see remark after Theorem 5.5), but let us examine a striking example of initial function which is not lower semi-continuous: we consider the characteristic function of the points with rational coordinates in $\mathbb{R}^{N}$, denoted by $v_{0}=\chi_{\mathbb{Q}^{N}}$. Then $v_{0}=0$ a.e., and it is clear that $\mathcal{Z}_{i}\left(v_{0}\right)=\mathbb{R}^{N}$, thus our modification yields $v_{0} \equiv 0$. In fact, the corresponding initial data $u_{0}$ is infinite almost everywhere, so that the associated solution $u$ is identically $+\infty$. Thus we obtain $v \equiv 0$, hence it is natural to consider re-defined initial data $v_{0} \equiv 0$.

Let us now state the complete existence and uniqueness theorem for equation (1.1).

Theorem 5.4 For every initial data $v_{0}$ in the class of measurable functions $\mathcal{L}^{+}$, every $\mathcal{S}$ satisfying (5.3), and every singular Radon measure $\mu_{s}$ supported in the complement of $\mathcal{S}$, there exists a unique solution of equation (1.1) in $\mathcal{W}_{s}$ which takes on the initial data $\nu=\left(\mathcal{S}, f, \mu_{s}\right)$ in the sense of $p$-trace with $p=-\gamma$. $\mathcal{S}$ is the zero set of $v$ for all $t>0$. Given $v_{0}$, the maximal solution corresponds to taking $\mu_{s}=0$ in the definition of $\nu$ and $\mathcal{S}=\mathcal{Z}_{i}\left(v_{0}\right)$. The rest of the assertions of Theorem 5.1 apply. 
Remark. It clearly appears that the problem of uniqueness is the freedom of choice of $\mathcal{S}$ and $\mu_{s}$, which gives rise to different $u$ 's, hence also different $v$ 's, with the same initial data $v_{0}$. The influence of $\mathcal{S}$ is reflected in the behaviour of the solution for all $t>0$ in the set of quadratic zeros, since $\mathcal{Z}_{0}(v)=\mathcal{S}$. The influence of $\mu_{s}$ is less obvious since the solutions become positive on the support of $\mu_{s}$, and this is a more delicate cause for problems.

Counterexample. The zero-level set of $v(t)$ has an important evolution property: a strong zero of $v_{0}$ persists in the solution $v \in \mathcal{W}_{s}$, while a weak zero becomes positive for any $t>0$. This is not true when we consider the larger class of solutions $\mathcal{W}$, where weak zeros may remain as such, as shows the example (where $c>0$ denotes a constant depending only on $N \geqslant 3$ ):

$$
u(x, t)=c|x|^{-\frac{N-2}{m}}, \quad v=c|x|^{(N-2) \frac{1-m}{m}} .
$$

The corresponding function $u$ satisfies $u_{t}=\Delta u^{m}+\delta_{0}(x)$, and has only a weak singularity at $x=0$, and $v$ satisfies the pressure equation although the zero at $x=0$ is not quadratic precisely if $m>(N-2) / N$.

VANISHING VISCOSITY. We have the following special case:

Theorem 5.5 The set of solutions obtained as limits of the vanishing viscosity method is precisely the set of maximal solutions, i.e., solutions where $v_{0} \in \mathcal{L}^{+}$is arbitrary, $\mathcal{S}=\mathcal{Z}_{i}\left(v_{0}\right)$ and $\mu_{s}=0$.

Proof. Given $v_{0}$ we use for the approximations $v_{0 \varepsilon}=v_{0}(x)+\varepsilon$ so that $u_{0 \varepsilon}$ is bounded and converges monotonically to $f$ a.e. $\left(v_{0}(x)=m f(x)^{m-1}\right)$. Hence, there is no measure $\mu_{s}$ and the only strong singular points are those generated by $f$, i.e., $\mathcal{S}=\mathcal{Z}_{i}\left(v_{0}\right)$.

Remark. In the particular case when $v_{0}$ is continuous and bounded, we recover the maximal viscosity solution of Bertsch et al., that is here uniquely characterized as the maximal solution in $\mathcal{W}_{s}$. If $v_{0}$ is not weakly lower semi-continuous, we have re-defined its zero-level set so that it becomes so. Another possibility is to make approximations of such a $v_{0}$ by initial functions in $\mathcal{L}_{s}^{+}$which are obtained by adding an $\varepsilon>0$ to $v_{0}$. This is equivalent to the vanishing viscosity method. Passage to the limit yields a solution $v$ such that for any $x_{0} \in \mathcal{Z}_{i}\left(v_{0}\right), v\left(x_{0}, t\right)=0$ for any $t>0$. However, if $v_{0}$ is not in $\mathcal{L}_{s}^{+}$, we may find such a point $x_{0}$ such that $v_{0}\left(x_{0}\right)>0$. The modification of the initial data that we made ensures that $v_{0}\left(x_{0}\right)=0$, which is rather natural. 


\section{Continuous initial data. Non-uniqueness results}

According to Theorems 5.1 and 5.4, the only reason for nonuniqueness of the Cauchy problem in the class of special weak solutions is the presence of the measures $\mathcal{S}$ and $\mu_{s}$ in the initial trace of $u \in T^{-1}(v)$. Let us examine the situation when the data are continuous. We then define the set of initial weak zeros as

$$
\mathcal{Z}_{w}\left(v_{0}\right)=\mathcal{Z}_{0}\left(v_{0}\right) \backslash \mathcal{S}
$$

Let us mention that necessarily $\mathcal{Z}_{w}$ if of zero Lebesgue measure in $\mathbb{R}^{N}$, otherwise there would be at least one intrinsic singular point in it. Indeed, if $\mathcal{Z}_{w}$ has positive measure, then $v^{-\gamma}$ is infinite on a set of positive measure, so that the integral of $v^{-\gamma}$ over any set containing $\mathcal{Z}_{w}$ is infinite, which is not possible unless the exists a strong zero in $\mathcal{Z}_{w}$. This is the same argument given in the proof of Proposition 5.3.

We first notice that the solutions constructed in Section 5 need not be continuous at $t=0$ because of the possible presence of a singular measure $\mu_{s}$ supported somewhere in the complement of $\mathcal{Z}_{0}$, or because of a set $\mathcal{S}$ chosen to contain points in the complement of $\mathcal{Z}_{0}$.

Proposition 6.1 If the initial function $v_{0}$ in Theorem 5.4 is continuous (resp. extended continuous), then there exists at least a solution $v$ with initial trace in $T^{-1}\left(v_{0}\right)$ that takes the initial data in the continuous (resp. extended continuous) sense, namely, the maximal solution. There are infinitely many that take the initial data a.e. but not continuously. A solution takes the initial data continuously if and only if the support of $\mu_{s}$ and $\mathcal{S}$ are contained in $\mathcal{Z}_{0}$.

Note that continuous initial data taken in the continuous way do not allow for the unique characterization of the solution. The following argument appears in [13]: at every point $x_{0}$ where $v_{0}\left(x_{0}\right)$ takes the value zero in the weak sense, the corresponding $u_{0}$ has a weak singularity at $x_{0}$, which is regularized for $t>0$ thanks to the $L_{l o c}^{1} \rightarrow L_{l o c}^{\infty}$ effect. However, we are free to add any finite Dirac mass at $x_{0}$. In this way, we generate an infinite amount of solutions $u$ such that $u(x, t)$ goes to infinity as $(x, t) \rightarrow\left(x_{0}, 0\right)$. This means that we will have infinitely many solutions $v(x, t)$ taking the same initial data in the continuous way. We point out that after our previous analysis, we can also add this point to the very singular set $\mathcal{S}$, thus obtaining a maximal solution in this class for $u$, which translates into a minimal solution for $v$. We recall that a minimal solution exists for continuous data under the requirement that these initial data are also taken continuously. We sum up these results as follows: 
Theorem 6.2 For every nonnegative and extended continuous initial function $v_{0}(x)$ which has weak zeros, there are infinitely many solutions $v \in \mathcal{W}_{s}$ of equation (1.1) taking $v_{0}$ as initial data in the continuous way. There is a maximal solution which has constant zero-level set consisting only of the strong zeros of $v_{0}, \mathcal{S}(t)=\mathcal{S}_{0}$, so that weak zeros become positivity points for $t>0$. There is also a minimal solution characterized by having $\mathcal{S}(t)=\mathcal{Z}_{0}$, so that all weak zeros become strong. Any intermediate closed set is allowed for $\mathcal{S}(t)$. Uniqueness holds when $\mathcal{Z}_{w}\left(v_{0}\right)=\emptyset$.

Now, even the knowledge of $\mathcal{S}(t)$ is not enough to characterize the solution when $\mathcal{S}(t) \neq \mathcal{Z}_{0}$. Indeed, in this case some weak zeros become positive which means that we did not add a strong singularity to $u_{0}$ at those points. But we can always add an arbitrary number of finite Dirac masses, $c_{i} \delta_{0}\left(x-x_{i}\right)$, thus producing different solutions $u$ 's, hence, different $v$ 's with the same zero-level set for $t>0$.

Remark. Existence of a minimal solution may be obtained under the weaker assumption " $v_{0}$ lower semi-continuous", since in that case the set $\left\{v_{0}>0\right\}$ is open, and $\mathcal{Z}_{0}$ is closed.

Sufficient assumptions in order to get only strong zeros, and then uniqueness may be obtained through the regularity of $v_{0}$. In particular, when the data are $C^{2}$, the $\mathcal{W}_{s}$ solution which takes the initial data continuously is unique, since in this case all zeros of $v_{0}$ are strong, i.e., $\mathcal{Z}_{0}=\mathcal{S}_{0}$. More precise conditions in the case of Hölder regularity are as follows:

Theorem 6.3 Let $v_{0} \geqslant 0$ in $\mathbb{R}^{N}$. We require the following condition

$$
\begin{aligned}
& v_{0} \in C^{1, N / \gamma-1}\left(\mathbb{R}^{N}\right) \quad \text { if } \quad N / 2<\gamma \leqslant N, \\
& v_{0} \in C^{0, N / \gamma}\left(\mathbb{R}^{N}\right) \quad \text { if } \quad N<\gamma<\infty .
\end{aligned}
$$

Then there exists a unique $v \in \mathcal{W}_{s}$ taking the initial data in the continuous sense.

Proof. We notice that under our assumptions, the corresponding initial data for the density variable $u, u_{0}=c v_{0}^{-\gamma}$ has only strong singularities. Indeed, if $v_{0}\left(x_{0}\right)=0$, then we have the behaviour $v_{0}(x)=O\left(\left|x-x_{0}\right|^{N / \gamma}\right)$ near $x=x_{0}$, hence

$$
u_{0}(x) \geqslant C\left|x-x_{0}\right|^{-N} \quad \text { near } \quad x=x_{0}, \quad C>0,
$$

which is not integrable. The strong zeros of $v_{0}$ remain as quadratic zeros at later times, and $v_{0}$ has no weak zeros, so that there is only one corresponding solution $v \in \mathcal{W}_{s}$, cf. Theorem 6.2. 
The exponents in the conditions are sharp, as one can see on the following explicit example, already seen as a counter-example to uniqueness of smooth solutions: Let us consider an initial $(-\gamma)$-trace of the form:

$$
u_{0, c}(x)=|x|^{-\alpha}+c \delta_{0},
$$

where $\alpha<N$ and $c>0$. Then the $u_{0, c}$ 's have a weak singularity at $x=0$, but with different masses according to $c>0$. Then every $c>0$ gives rise to a corresponding solution $v_{c}$ taking the $(-\gamma)$-trace $u_{0, c}$. Hence, all the $v_{c}$ 's are different, but they take on the same initial data $v_{0}(x)=m|x|^{\alpha / \gamma}$ continuously. Moreover, $v_{0}$ is in $C^{0, \alpha / \gamma}$ or $C^{1, \alpha / \gamma-1}$, according to $\gamma$. Since $\alpha$ may be arbitrary close to $N$, this proves the sharpness of the exponents. Note that for $\alpha \geqslant N$, the singularity at $x=0$ is always strong, whatever $c>0$, hence the $v_{c}$ 's are identical.

In particular, when we take an initial function $v_{0} \in C^{0}\left(\mathbb{R}^{N}\right)$ in these Hölder classes that has only weak zeros we conclude that there exist infinitely many classical solutions $v \in \mathcal{C}_{q}$ which are positive, hence $C^{\infty}$ smooth for $t>0$ and take the initial data $v_{0}$ is a continuous way.

\section{Non-existence for initial measures}

The existence result, Theorem 4.6, cannot be extended to include measures with non-zero singular part (with respect to the Lebesgue measure) as initial data for $v$, if we want to preserve usual properties of the solutions, like some weak continuous dependence of the solutions on the data. The reason is that in any approximation, the singular part of the measure will tend to zero for the fast-diffusion variable $u$. According to the theory for this equation, it will not be seen in the limit because "we lose its trace". We give next a precise result for Radon measures.

Proposition 7.1 Let $\mu$ be a Radon measure in $\mathbb{R}^{N}$ which we write as

$$
d \mu=f(x) d x+d \mu_{s},
$$

where $f \in L_{l o c}^{1}\left(\mathbb{R}^{N}\right)$ and $\mu_{s}$ is singular with respect to the Lebesgue measure. Let $\mu_{n}$ be any approximation by $L_{l o c}^{1}$ functions of $\mu_{s}$ and $v_{n}$ be the unique solution in $\mathcal{W}_{s}$ corresponding to the data $f+\mu_{n}$. Then $v_{n}$ converges to the unique solution $v_{f}$ in $\mathcal{W}_{s}$ with initial $p$-trace $c f^{-\gamma}, p=-\gamma$. In other words, the singular part of the measure disappears in the limit.

Proof. Let us first consider the case where $f \geqslant \varepsilon$ for some $\varepsilon>0$. We pass to equation (1.3) and consider the initial data $u_{n 0}=c\left(f+\mu_{n}\right)^{-\gamma}$, dominated by $g=c f^{-\gamma} \leqslant c \varepsilon^{-\gamma}$. Thus, by the results of [36], it is clear that the corresponding solution $u_{n}$ takes the initial data in $L_{l o c}^{1}\left(\mathbb{R}^{N}\right)$. 
Moreover, since $\mu_{n} \rightarrow 0$ almost everywhere, we have by dominated convergence,

$$
u_{n 0} \rightarrow c f^{-\gamma} \quad \text { in } \quad L_{l o c}^{1}\left(\mathbb{R}^{N}\right)
$$

Then, by well-posedness of the Cauchy problem for $u$,

$$
u_{n}(t) \rightarrow u(t) \quad \text { locally uniformly in } Q
$$

where $u$ is the unique continuous solution of equation (1.3) with initial data $u_{0}$. Thus, $v_{n}$ converges locally uniformly to the unique solution $v \in \mathcal{W}_{s}$ associated with $u$, which has $(-\gamma)$-trace $c f^{-\gamma}$.

When $f$ is only supposed to be nonnegative, we consider the sequence of solutions $v_{\varepsilon, n} \in \mathcal{W}_{s}$ with initial data

$$
v_{\varepsilon, n}(0)=f+\varepsilon+\mu_{n}, \quad \varepsilon>0 .
$$

Let us call $v_{f}$ the unique solution in $\mathcal{W}_{s}$ with $(-\gamma)$-trace $c f^{-\gamma}$ and $v_{n}$ the unique solution in $\mathcal{W}_{s}$ with $(-\gamma)$-trace $c\left(f+\mu_{n}\right)^{-\gamma}$. Again by well-posedness in the density variable $u$, it is clear that the following comparison result holds:

$$
v_{f}(x, t) \leqslant v_{n}(x, t) \leqslant v_{n, \varepsilon}(x, t) \text { in } Q .
$$

Now, by the first part of the proof, we know that when $n \rightarrow \infty, v_{n, \varepsilon}$ converges to the unique solution $v_{f+\varepsilon} \in \mathcal{W}_{s}$ with $(-\gamma)$-trace $c(f+\varepsilon)^{-\gamma}$. Moreover, when $\varepsilon$ decreases to zero, it is easy to see that $v_{\varepsilon}$ decreases to $v_{f}$, by uniqueness in the density variable since these solutions have the same $(-\gamma)$-trace. Thus we obtain

$$
v_{n}(x, t) \rightarrow v_{f}(x, t) \quad \text { locally uniformly in } Q
$$

hence the result.

The same proof applies for a Borel measure such that the singular set of $v_{0}, \mathcal{S}_{v_{0}}$, has measure zero, or to any of its connected components which has measure zero. On the other hand, if the measure of $\mathcal{S}_{v_{0}}$ is positive, there is a solution that sees this singular initial value in the following sense:

Proposition 7.2 For every density point of the set $\mathcal{S}_{v_{0}}$, we have

$$
\lim _{t \rightarrow 0} \int_{B_{r}\left(x_{0}\right)} v(x, t)=+\infty,
$$

where $v$ is the solution of equation (1.1) obtained as monotone limit of solutions with bounded initial data $v_{0 n}$ that converge to $v_{0}$. 
Proof. A point of density of a set $E$ is a point $x_{0} \in$ such that for every $r>0$, we have $\left|B_{r}\left(x_{0}\right) \cap E\right|>0$. Then by approximation we can find for any $c$ large enough

$$
\int_{B_{r}\left(x_{0}\right)} v_{n}(x, t) \geqslant c
$$

if $n \geqslant n_{0}$ and $0<t<t_{0}(n, c)$, since the initial data are taken in $L_{l o c}^{1}$ (see next section). Then,

$$
\lim _{t \rightarrow 0} \int_{B_{r}\left(x_{0}\right)} v(x, t) \geqslant \lim _{t \rightarrow 0} \int_{B_{r}\left(x_{0}\right)} v_{n}(x, t)=+\infty .
$$

\section{Trace in the $L_{l o c}^{1}$-sense}

If the initial data are not continuous, we know that almost everywhere convergence always holds. This is interesting information, but we would like the initial data to be taken in a somewhat better sense. We give below sufficient conditions on the initial data in order to get a trace in the "classical" $L_{l o c}^{1}$ sense.

Theorem 8.1 Let $\gamma>\max \{N / 2,1\}$ and $v_{0} \in L^{\infty}\left(\mathbb{R}^{N}\right)+L^{1}\left(\mathbb{R}^{N}\right)$ nonnegative. Then for any $v \in \mathcal{W}_{s}$ with $(-\gamma)$-trace $u_{0} \in T^{-1}\left(v_{0}\right)$, the initial data are taken as standard trace in $L_{l o c}^{1}\left(\mathbb{R}^{N}\right)$ :

$$
\lim _{t \rightarrow 0} v(x, t)=v_{0}(x) \quad \text { in } \quad L_{l o c}^{1}\left(\mathbb{R}^{N}\right) .
$$

Proof. By uniqueness in $\mathcal{W}_{s}$ (thanks to the concept of $(-\gamma)$-trace), we may use approximations of the solution $v$. Let $v_{n}$ be a smooth solution with initial data $v_{0 n} \in C^{0}\left(\mathbb{R}^{N}\right) \cap L^{\infty}\left(\mathbb{R}^{N}\right)$ such that

$$
v_{0 n} \rightarrow v_{0} \quad \text { in } \quad L^{\infty}\left(\mathbb{R}^{N}\right)+L^{1}\left(\mathbb{R}^{N}\right),
$$

and in the sense of $(-\gamma)$-trace. Then we shall make estimates of $v_{n}$ in $L^{2}\left(0, T ; H_{l o c}^{1}\left(\mathbb{R}^{N}\right)\right)$ to pass to the limit in the weak formulation. If we multiply the equation by $p(v)$, where

$$
p(x)=\left\{\begin{array}{cc}
0 & \text { if } x \leqslant k \\
x-k & \text { if } k<x<k+1, \\
1 & \text { if } x>k
\end{array}\right.
$$

for some $k>0$, and integrate by parts, we find:

$$
\int j\left(v_{0 n}\right)=\int j\left(v_{n}\right)(t)+(\gamma+1) \int_{0}^{t} \int\left|\nabla v_{n}\right|^{2} p\left(v_{n}\right)+\int_{0}^{t} \int v_{n} p^{\prime}\left(v_{n}\right)\left|\nabla v_{n}\right|^{2},
$$

where $j$ is the primitive of $p$ such that $j(0)=0$. 
Since we have assumed that $v_{0 n}$ converges to $v_{0}$ in $L^{\infty}+L^{1}$, for $k$ large enough there exists a constant $C>0$ such that $\int j\left(v_{0 n}\right) \leqslant C$, which proves that when $n \rightarrow \infty, v_{n}(t)$ remains uniformly bounded in $L^{\infty}+L^{1}$, and moreover,

$$
\int_{0}^{t} \int_{u \geqslant k}\left|\nabla v_{n}\right|^{2} \leqslant C .
$$

Now let us take $\varphi \in C_{0}^{\infty}\left(\mathbb{R}^{N}\right)$, and multiply this time by $p(v) \varphi$ which yields:

$$
\begin{aligned}
\int j\left(v_{0 n}\right) \varphi= & \int j\left(v_{n}\right)(t) \varphi+(\gamma+1) \int_{0}^{t} \int\left|\nabla v_{n}\right|^{2} p\left(v_{n}\right) \varphi+\int_{0}^{t} \int v_{n} p^{\prime}\left(v_{n}\right)\left|\nabla v_{n}\right|^{2} \varphi \\
& +\int_{0}^{t} \int v_{n} p\left(v_{n}\right) \nabla v_{n} \nabla \varphi .
\end{aligned}
$$

The last term can be written:

$$
\int_{0}^{t} \int v_{n} p\left(v_{n}\right) \nabla v_{n} \nabla \varphi=\int_{0}^{t} \int \nabla l\left(v_{n}\right) \nabla \varphi=-\int_{0}^{t} \int l\left(v_{n}\right) \Delta \varphi
$$

where $l(s)$ is the primitive of the function $s p(s)$ such that $l(0)=0$. Then we take an approximation of the solution of the problem

$$
-\Delta \varphi=\rho \in C_{0}^{\infty}\left(\mathbb{R}^{N}\right), \quad 0 \leqslant \rho \leqslant 1,
$$

and pass to the limit since $\varphi$ is bounded. By the previous estimates,

$\int j\left(v_{n}\right)(t) \varphi+(\gamma+1) \int_{0}^{t} \int\left|\nabla v_{n}\right|^{2} p\left(v_{n}\right) \varphi+\int_{0}^{t} \int v_{n} p^{\prime}\left(v_{n}\right)\left|\nabla v_{n}\right|^{2} \varphi+\int_{0}^{t} \int l\left(v_{n}\right) \rho \leqslant C$.

This proves that $l\left(v_{n}\right)$ remains bounded in $L^{1}\left(0, T ; L_{l o c}^{1}\left(\mathbb{R}^{N}\right)\right)$, hence $v_{n}$ remains bounded in $L^{2}\left(0, T ; H_{l o c}^{1}\right)$. Thus, there exist a subsequence still noted $v_{n}$ such that

$$
\begin{aligned}
v_{n} & \rightarrow v \quad \text { in } \quad L^{2}\left(0, T ; L_{l o c}^{2}\left(\mathbb{R}^{N}\right)\right) \text { strong, } \\
\nabla v_{n} & \rightarrow \nabla v \quad \text { in } \quad L^{2}\left(0, T ; L_{l o c}^{2}\left(\mathbb{R}^{N}\right)\right) \text { weak. }
\end{aligned}
$$

Indeed, since we have assumed that the initial data converge also in the sense of $(-\gamma)$-trace, we can use the well-posedness property of the density problem which proves that the limit is exactly $v$. This is enough to pass to the limit in the weak formulation, which gives that for every $\varphi \in C^{2}\left(\mathbb{R}^{N} \times[0, T)\right)$, with fixed compact support in $x$ :

$$
\int v(t) \varphi-\int_{0}^{t} \int v \varphi_{t}-\int_{0}^{t} \int v \nabla v \nabla \varphi+(\gamma+1) \int_{0}^{t} \int|\nabla v|^{2} \varphi=\int \varphi v_{0} .
$$


This proves also that

$$
\int v(t) \varphi \underset{t \rightarrow 0}{\longrightarrow} \int \varphi v_{0}
$$

but since $v(t) \rightarrow v_{0}$ almost everywhere, it follows that the initial data is taken in $L_{l o c}^{1}$ :

$$
\int_{K}\left|v(t)-v_{0}\right| \underset{t \rightarrow 0}{\longrightarrow} 0
$$

Indeed, by dominated convergence, $\int_{K}\left(v_{0}-v(t)\right)_{+} \rightarrow 0$, while writing

$$
\int_{K}\left(v_{0}-v(t)\right)_{-}=\int_{K} v_{0}-\int_{K} v(t)+\int_{K}\left(v_{0}-v(t)\right)_{+},
$$

we deduce that also the negative part of $v_{0}-v(t)$ converges in $L_{l o c}^{1}$. Then clearly (8.1) holds.

Remark. By a small modification of the construction, one can show that in fact existence holds if the initial data is in $L^{\infty}\left(\mathbb{R}^{N}\right)+L^{1}\left(\mathbb{R}^{N}, \rho d x\right)$, where the weight behaves like the Newton kernel at infinity:

$$
\rho(x) \underset{|x| \rightarrow \infty}{\sim}|x|^{2-N}
$$

if $N>2$, and the logarithm if $N=2$.

Continuous dependence. It is clear first that we have continuous dependence on the initial data in the sense of $(-\gamma)$-trace (by uniqueness). Now, the almost everywhere convergence of the data is not sufficient to ensure continuous dependence on the initial data, not even the $L_{l o c}^{p}$ convergence, as we prove below:

Proposition 8.2 There exists a sequence $v_{0 n} \in C^{0}\left(\mathbb{R}^{N}\right)$ with the property that $v_{0 n} \rightarrow 1$ in $L_{l o c}^{p}\left(\mathbb{R}^{N}\right)$ for all $1 \leqslant p<\infty$, and $v_{n}$ converges locally uniformly to some $v$ in $\mathbb{R}^{N} \times(0, \infty)$. But $v \not \equiv 1$, which is the unique solution in $\mathcal{W}_{s}$ with $(-\gamma)$-trace $\nu \equiv 1$.

Proof. Let $\rho_{n} \geqslant 0$ be an approximation of $\delta_{0}$ and consider the initial data $v_{0 n}=c\left(1+\rho_{n}\right)^{-1 / \gamma}$. Then clearly $v_{0 n} \rightarrow 1$ almost everywhere and for any $n \in \mathbb{N}, 0 \leqslant v_{0 n} \leqslant 1$, so that indeed, $v_{0 n} \rightarrow 1$ in any $L_{l o c}^{p}\left(\mathbb{R}^{N}\right), 1 \leqslant p<\infty$. We know that for any $n \in \mathbb{N}$, there exists a unique $v_{n} \in \mathcal{W}_{s}$ which takes on the initial data in the sense of $(-\gamma)$-trace (and in fact continuously). Now if $u_{n}=c v_{n}^{-\gamma}$, then $u_{n}$ takes on the initial trace

$$
u_{0 n}=1+\rho_{n},
$$


so that when $n \rightarrow+\infty$, by well-posedness in the class $\mathcal{E}_{c}, u_{n}$ will converge to the unique $u \in \mathcal{E}_{c}$ which has initial trace $u_{0}=1+\delta_{0}$. Thus, $v_{n} \rightarrow v=c u^{-1 / \gamma}$, which is not identically 1 .

However, if we assume monotonicity then continuous dependence holds since this implies convergence in $(-\gamma)$-traces: if $v_{0 n}$ converges monotonically to $v_{0}$, then any $\nu_{n} \in T^{-1}\left(v_{0 n}\right)$ will converge monotonically to some $\nu \in$ $T^{-1}\left(v_{0}\right)$, so that for any $\varphi \in C_{0}, \varphi \geqslant 0$,

$$
\int_{\mathbb{R}^{N}} c v_{0 n}^{-1 / \gamma} \varphi \underset{n \rightarrow \infty}{\longrightarrow} \int_{\mathbb{R}^{N}} c v_{0}^{-1 / \gamma} \varphi \text { monotonically, }
$$

whether the integral is finite or not.

\section{Special solutions and asymptotic behaviour}

We divide this section into three paragraphs concerning different types of solutions and asymptotic behaviours.

\section{Separable Solutions and Asymptotics in $\mathcal{W}_{s}$}

We study here the asymptotic behaviour for the solutions of (1.1), in the case of constant zero-level set. In the sequel we will recall the results from [21] and translate them to equation (1.1).

Analogously to the notation $u_{(\mathcal{S}, \mu)}$ for the E.C.S. of equation (1.3) with initial trace $(\mathcal{S}, \mu)$, we denote by $v_{\left(\mathcal{S}, v_{0}\right)}$ the unique solution of $(1.1)$ in $\mathcal{W}_{s}$ with initial data

$$
v_{\left(\mathcal{S}, v_{0}\right)}(x, 0)=\left\{\begin{array}{cll}
0 & \text { if } & x \in \mathcal{S} \\
v_{0}(x) & \text { if } & x \notin \mathcal{S}
\end{array}\right.
$$

It is interesting to notice that if $\mathcal{S}$ is empty and $v_{0}$ is bounded away from zero, then the corresponding solution $v \in \mathcal{W}_{s}$ will also remain bounded away from zero. On the contrary, if $v_{0}$ has a strong zero, say, at $x=x_{0}$, then we have seen that the following estimate holds:

$$
v(x, t) \leqslant C \frac{\left|x-x_{0}\right|^{2}}{t},
$$

which proves that $v$ goes to zero as $t \rightarrow \infty$, locally uniformly in $\mathbb{R}^{N}$. This means that strong zeros behave like permanent sinks, a first important information about asymptotic behaviour. We also have a first rate, which turns out to be accurate.

We have seen in Section 2 examples of explicit solutions of our problem. The next class of separable solutions can be obtained as a direct consequence of the analysis in [21]. 
Theorem 9.1 Corresponding to data $(\mathcal{S}, \infty)$ there is a unique separable solution of the form

$$
v_{(\mathcal{S}, \infty)}=t^{-1} g(x)
$$

where $g=g_{\mathcal{R}}(x)$ is the unique solution of the equation

$$
g \Delta g-\gamma|\nabla g|^{2}+g=0
$$

which is defined and positive in $\mathcal{R}=\mathbb{R}^{N} \backslash \mathcal{S}$ and has strong zeros at the boundary. This function is extended continuously to the whole space by setting $g=0$ on $\mathcal{S}$.

We emphasize that there is no restriction whatsoever on the closed set $\mathcal{S}$ or its complement $\mathcal{R}$, cf. [21]. The case $\mathcal{S}=\{0\}$ gives rise to the quadratic solution $(2.3)$. In $[44,38]$ the profile $g$ is calculated in one dimension when $\mathcal{R}$ is the interval $I=(-l, l)$ and $\gamma=1$ as

$$
g(x)=\frac{a^{2}}{2} \cos ^{2}\left(\frac{x}{a}\right), \quad a=\frac{2 l}{\pi} .
$$

Solutions taking infinite initial data on large sets may seem strange at first sight, but they are natural in our problem, simple to construct, and represent the asymptotic behaviour of large classes of solutions, as we will see next.

Theorem 9.2 Let $v_{\left(\mathcal{S}, v_{0}\right)} \in \mathcal{W}_{s}$ be the solution with initial data defined by (9.1). Then,

$$
\lim _{|x| \rightarrow+\infty}|x|^{-2} v_{\left(\mathcal{S}, v_{0}\right)}(x, t) \leqslant \frac{\kappa}{t} .
$$

This rate comes from the estimate for the E.C.S. of equation (1.3) with initial trace $(\mathcal{S}, \mu)$, which reads

$$
\lim _{|x| \rightarrow \infty}|x|^{2 /(1-m)} u_{(\mathcal{S}, \mu)}(x, t) \geqslant(C t)^{\frac{1}{1-m}} .
$$

This rate is optimal and minimal for $u$, and moreover the limit is exact in the case of compactly supported initial traces. Therefore, (9.4) is maximal and optimal for initial $v_{0}$ which is infinity in the complement of a ball. But we warn the reader that the result is deceptive. Though it says that the limit quotient $($ as $x \rightarrow \infty) v(x, t) /|x|^{2}$ goes to zero as $t \rightarrow \infty$, this does not mean that $v(x, t)$ goes to zero at any given $x$, as the Barenblatt solutions (2.5) show (actually, the limit is infinite at every fixed $x$ ). Such a behaviour is 
restricted to certain solutions, in particular the ones having strong zeros. For these more can be said. The following limit holds locally uniformly in $\mathcal{R}=\mathbb{R}^{N} \backslash \mathcal{S}$ :

$$
\lim _{t \rightarrow \infty} t^{-\frac{1}{1-m}}\left|u_{(\mathcal{S}, \mu)}-u_{(\mathcal{S}, 0)}\right|(x, t)=0 .
$$

From this limit we get the expansion $u_{(\mathcal{S}, \mu)}=u_{(\mathcal{S}, 0)}+o\left(t^{1 /(1-m)}\right)$, which yields immediately the following transcription in terms of $v$ :

Theorem 9.3 Locally uniformly in $x$ we have

$$
\lim _{t \rightarrow+\infty} t v_{\left(\mathcal{S}, v_{0}\right)}(x, t)=g_{\mathcal{R}}(x) .
$$

The limit is uniform if $\mathcal{R}$ is bounded.

This result is proved in [38] in the particular case $N=1, \gamma=1$ and $\mathcal{R}$ is an interval. It is true also for $\gamma<N / 2$ but we will not discuss that issue.

A further result from [21] concerns intermediate asymptotic behaviour in expanding sets and reads as follows. Let $\beta>0$ and let us assume that $u$ is the E.C.S. with initial trace $\nu=(\mathcal{S}, \mu)$, where $\mathcal{S}$ is bounded and non-empty, and $d \mu=f d x$. Under the condition

$$
f(x)=o\left(|x|^{p}\right), \quad p=\frac{1-2 \beta}{\beta(1-m)},
$$

we have the asymptotic formula

$$
\lim _{t \rightarrow \infty} t^{-\alpha}\left|u(y, t)-U_{\infty}(y, t)\right|=0, \quad \alpha=\frac{1-2 \beta}{1-m}>0
$$

uniformly on sets of the form $C_{1} t^{\beta} \leqslant|y| \leqslant C_{2} t^{\beta}$. From this, we deduce a similar intermediate asymptotics result for $v$ :

Theorem 9.4 Under the condition that $\mathcal{S}$ is bounded and non-empty and that $v_{0}$ behaves at infinity in the form

$$
\lim _{|x| \rightarrow \infty} v_{0}(x)|x|^{(1-2 \beta) / \beta}=\infty,
$$

we have

$$
\lim _{t \rightarrow \infty} t^{1-2 \beta}\left|v(x, t)-V_{0}(x, t)\right|=0,
$$

on sets of the form $C_{1}|y|^{\beta} \leqslant|y| \leqslant C_{2}|y|^{\beta}$, where $V_{0}$ is the explicit quadratic solution (2.3). 
The proof can be performed by copying the rescaling analysis of [21], using this time the scaling transformation

$$
v_{\lambda}(x, t)=\lambda^{\mu} v\left(\lambda^{\beta} x, \lambda t\right)
$$

with $\mu=1-2 \beta$. If $v$ is the solution, then $v_{\lambda}$ is again a solution of (1.1) and we let $\lambda \rightarrow \infty$ to get the result as in [21], Theorem 7.5.

II. Stationary Solutions. As was already seen, for $\gamma \neq 1$ there exist stationary solutions of the form

$$
v_{*}(x, t)=C|x|^{\sigma}, \quad \sigma=\frac{N-2}{\gamma-1}, \quad C>0 .
$$

The behaviour depends strongly on the space dimensions

- Case $N \geqslant 3$ :

- If $\gamma>N / 2$ we have $\sigma \in(0,2)$, so that $v_{*}$ is never in $\mathcal{C}_{q}$. It lies in $\mathcal{C}$ whenever $\gamma<N-1$. It has a permanent weak zero, and the solution in $\mathcal{C}_{q}$ with these initial data is everywhere positive in $\mathbb{R}^{N} \times(0, \infty)$.

- If $1<\gamma \leqslant N / 2, v_{*}$ is strictly classical, i.e., $v \in C^{2}$ and has a quadratic zero. Here, $\sigma \geqslant 2$.

- For $\gamma=1$ the radially symmetric stationary solution reads

$$
v_{*}(x, t)=C \exp \left(-|x|^{-(N-2)}\right)
$$

which is a strictly classical solution with a zero beyond all powers.

- For $0<\gamma<1$ we get stationary solutions with a singularity at $x=0$, away from our classical or weak theories.

Other solutions can be obtained from the fast-diffusion formulation where we get $\Delta u_{*}^{m}=0$, hence

$$
u_{*}^{m}(x, t)=C|x|^{2-N}+C_{1} .
$$

and we replace $u^{m}$ by $\log u$ when $m=0$. These new solutions do not offer essential novelties.

- Case $N=1$ : Arguing in the same way as for $N \geqslant 3$ the discussion is now very different, since $N-2=-1<0$.

- If $0<\gamma<1 / 2, v_{*}$ is a weak solution with a permanent weak zero at $x=0$.

- If $1 / 2 \leqslant \gamma<1, v_{*}$ is strictly classical i.e., $v_{*} \in C^{2}$, and the zero at $x=0$ is quadratic.

- If $\gamma>1: v_{*}$ has a standing singularity at $x=0$ since $\sigma=-1 /(\gamma-1)<0$. The singularity is strong, i.e., $v_{*}$ is not integrable near $x=0$ if and only if $\gamma \leqslant 2$. 
- Case $N=2$ : From the formula $u_{*}^{m}(x, t)=C \log (1 /|x|)$, we get

$$
v_{*}(x, t)=C\left(\log \left(\frac{1}{|x|}\right)\right)_{+}^{\frac{1}{1-\gamma}}
$$

if $\gamma \neq 1$, while for $\gamma=1, m=0$ we have $\log (u)=C \log (1 /|x|)+C_{1}$ and

$$
v_{*}(x, t)=C_{1}|x|^{C},
$$

with arbitrary constants $C, C_{1}$. Therefore, all of the above described situations happen now at $\gamma=1$. Indeed, it is well known that this equation has special properties, cf. [31].

- For $\gamma<1$ we get a solution in the unit ball with a singularity at $x=0$ and zero boundary value at $|x|=1$ with behaviour $v \sim d(x)^{1 /(1-\gamma)}$, which is quadratic only if $\gamma \geqslant 1 / 2$. It is a weak solution for all $0<\gamma<1$. On the other hand, for $\gamma>1$ we have singularities at the boundary.

III. Self-Similar Solutions. Finally, we consider the class of self-similar solutions, of the form

$$
v(x, t)=t^{-\mu} G\left(x t^{-\beta}\right), \quad \mu=1-2 \beta,
$$

which can be obtained for every $\beta \in \mathbb{R}$. We refer to [21], Appendix, for the equivalent analysis in terms of the density variable. The following conclusions are drawn upon translation to the pressure variable.

(i) The initial data can be chosen to behave like a power of $x$,

$$
v_{0}(x)=c|x|^{\sigma}, \quad \sigma=-\frac{\mu}{\beta}=2-\frac{1}{\beta}, \quad c>0,
$$

but for the choice $\beta=\theta / N=\gamma /(2 \gamma-N), \sigma=N / \gamma$, where we typically obtain the Barenblatt solutions $V_{A}(x, t)$ which have initial data infinite for all $x \neq 0$.

(ii) For $0<\beta \leqslant \theta / N, \sigma \geqslant N / \gamma$, we can choose a self-similar solution that becomes positive at the origin for all $t>0$ and then it behaves like

$$
v \sim t^{-\mu} \quad \text { as } t \rightarrow \infty
$$

uniformly on compact sets of $x$. The limit $\beta=0$ is the separable case.

(iii) For $\beta>\theta / N$ we get necessarily solutions with a strong zero at $x \neq 0$. In the limit $\beta=\infty$ we have $\sigma=2$ and we obtain the quadratic solutions.

(iv) For $\beta<0$ we get $\sigma>2$ and we have solutions with a very strong zero at the origin (larger than quadratic rate), whose local shape is conserved in time. 
We can use these results to obtain the asymptotic behaviour of solutions that have a growth at infinity of the form $v \sim|x|^{\sigma}$. The solution is then shown to decay in time (or grow if $\mu<0$ ) like $O\left(t^{-\mu}\right)$ on compact sets of $x$ if $\beta \leqslant \theta / N$, but it has necessarily a strong zero at $x=0$ if $\beta>\theta / N$. We skip the lengthy details for questions of space and refer to [21].

On the other hand, Kamin and Dascal [38] show how to use renormalization of the separable solution for data which decay exponentially. In that case they obtain a decay of the type

$$
v(x, t) \sim \frac{(\log t)^{2}}{t} \text { as } t \rightarrow \infty
$$

which is a "small" correction of the optimal $1 / t$ rate of the quadratic solution. Their result can be generalized to several space dimensions in the framework of the present paper.

\section{The Cauchy-Dirichlet Problem}

In this section, we add some remarks on the following Cauchy-Dirichlet problem in any open subset $\Omega \subset \mathbb{R}^{N}$ :

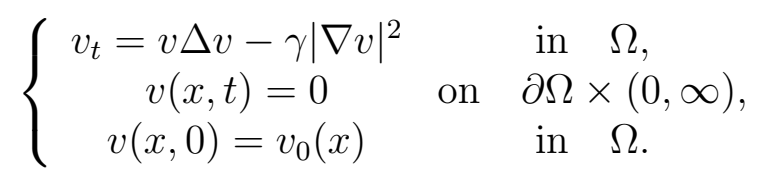

Actually, this problem may be seen as a particular case of the Cauchy Problem by requiring that $v=0$ outside $\Omega$. Thus we have the following immediate Corollary of Theorem 5.4:

Corollary 10.1 For any $v_{0} \in \mathcal{L}^{+}(\Omega)$, any closed set $\mathcal{S}$ containing $\mathcal{Z}_{i}\left(v_{0}\right)$ and $\mathbb{R}^{N} \backslash \Omega$, and any nonnegative singular measure $\mu_{s}$ supported in the complement of $\mathcal{S}$, there exists a unique solution in $\mathbb{R}^{N} \times(0, \infty) v \in \mathcal{W}_{s}$ which has $(-\gamma)$-trace $\nu=\left(\mathcal{S}, f, \mu_{s}\right), f$ defined as usual. Then $v$ is identically zero outside $\Omega$, so that it yields a solution in $\Omega \times(0, \infty)$.

$\mathcal{S}$ is the constant zero-level set of $v$, which also takes on the zero boundary data in the classical sense. The maximal solution corresponds to the choice $\mu_{s}=0$ and $\mathcal{S}=\mathcal{Z}_{i}\left(v_{0}\right) \cup\left\{\mathbb{R}^{N} \backslash \Omega\right\}$.

Note that in this result, we assume nothing on the regularity or the boundedness of $\Omega$, provided it is open. Of course, the maximal solution may be obtained by the vanishing viscosity method in $\Omega$, and the classes $\mathcal{W}_{s}$ and $\mathcal{C}_{q}$ are the same. Moreover, all the results concerning continuous data and the problems of uniqueness as well as nonexistence for singular measures (Sections 6 and 7) hold with obvious adaptations. 


\section{About the classes of contracting and expanding so- lutions}

When we consider solutions in the larger class $\mathcal{W}$, there is the possibility of working with solutions with an expanding zero-level set, i.e., the support of the solution contracts and may even vanish completely. Bertsch et al. [13] have shown that corresponding to continuous and compactly supported initial data there exist infinitely many solutions with different shrinking supports and they may even vanish identically in finite time. But the class offers also some possibilities of solutions with expanding supports, which are not in $\mathcal{C}_{q}$.

Contracting supports. The simplest solutions with contracting supports are the traveling waves, exhibited in Section 2. The next well-known examples of solutions of this type are the modifications of the quadratic solution (2.3), which behave differently for $0<\gamma<N / 2$ and $\gamma>N / 2$. In the latter case, preferred in this work, we have the so-called Barenblatt sourcetype solutions and the pseudo-Barenblatt family which have been described in Section 2. We still have another related family,

$$
V(x, t)=\frac{\left(B(T-t)^{2 \theta / N}-k|x|^{2}\right)_{+}}{T-t},
$$

which represents complete extinction in finite time with an interface that shrinks to a point.

The situation is somewhat different for $0<\gamma<N / 2$ though the property of contracting supports with linear behaviour stays. The quadratic solution stays the same. We have the explicit solutions with moving interfaces

$$
V(x, t)=\frac{\left(A t^{2 \theta / N}-k_{1}|x|^{2}\right)_{+}}{t}, \quad \theta=\frac{N \gamma}{N-2 \gamma},
$$

with $k_{1}=1 /(2(N-2 \gamma))$, and

$$
V(x, t)=\frac{k_{1}|x|^{2}+A(T-t)^{2 \theta / N}}{T-t} .
$$

All these solutions are examples of weak solutions or classical free-boundary solutions with a self-similar form and most have appeared in the previous references. In all the examples the behaviour near the zero-level set is not quadratic as in (4.8) but linear, a characteristic of moving interfaces.

A detailed study of the one-dimensional problem with moving interfaces is performed in [51], where the relation of moving interfaces with the socalled Darcy's law is examined. 
ExPANDING SUPPORTS. A basic example of this type of solutions is the following. Let us consider in dimension $N \geqslant 2$ the solution $u$ of the problem

$$
\left\{\begin{array}{cl}
u_{t}-\Delta u^{m}=\delta_{0}(x) \otimes f(t) & \text { in } Q \\
u_{0}(x)=1 & \text { in } \mathbb{R}^{N}
\end{array}\right.
$$

where $f \in C^{0}([0, \infty))$ is positive in $(0, \tau)$, and zero in $(\tau, \infty)$. This solution is constructed in [21]. Then the support of the corresponding $v=m u^{m-1}$ is exactly $\mathbb{R}^{N} \backslash\{0\}$ for $t \in(0, \tau)$ and the whole space $\mathbb{R}^{N}$ for later times, thus $v$ has an expanding support.

Many other such solutions are obtained from translation of the results in [20], where $f$ may be replaced by any Radon measure on $(0, \infty)$. In particular, if $f$ has a zero-level set with different connected components, the support of $v$ will "oscillate": if we take for instance

$$
f(t)=\max \{\sin (t), 0\}
$$

then the support of $v$ is $\mathbb{R}^{N} \backslash\{0\}$ for $t \in[2 k \pi,(2 k+1) \pi](k \in \mathbb{N})$, and the whole space $\mathbb{R}^{N}$ for other times. This will be a weak solution, but it will not be continuous at the points where the new interval of positivity of $f(t)$ starts, since $v(0, t)$ passes from a positive value to zero. But a continuous solution is obtained if we consider for instance

$$
f(t)=|\sin (t)|
$$

In general, we have to take into account that if we want to have continuous solutions then we need to have a weak zero at the initial time and $f(t)$ has to be positive in an interval, or have isolated zeros in it. We also recall that a strong zero of $v_{0}$ will not do, as it will remain for all later times as a quadratic zero.

Another type of solutions is obtained when we consider measures in the right-hand member supported in different points, like

$$
\sum_{j} \delta_{0}\left(x-x_{j}\right) \otimes f_{j}(t)
$$

the sum being finite or infinite.

As a conclusion, since the information about the measures in the righthand side, like $\delta_{0}(x) \otimes f(t)$ in the first example, disappears from the definition of the solution as a classical solution, there is no way we can identify or compare solutions in the class $\mathcal{C}$ by their initial data. 


\section{Conclusions and comments}

We have succeeded in establishing well-posedness of the Cauchy problem for the pressure equation (1.1) in the range $\gamma>N / 2$ in the class of special classical solutions $\mathcal{C}_{q}$, when we are given as initial data any measurable nonnegative function, finite or infinite, plus some additional information on the behaviour of the solution near the initial zeros that is coded as $p$-trace with $p=-\gamma$. We have shown that these solutions can also be characterized as the class of special weak solutions. They can be obtained as limits of smooth solutions of approximate problems, but not all of them are limits of the standard vanishing viscosity approximation.

Sinks. An interesting consequence of the results in view of the applications to biological diffusion is the following: while the intrinsic zeros $\mathcal{Z}_{i}$ of the initial function $v_{0}$ always lead to a solution $v$ (a concentration) which vanishes at these points for all times (fixed sinks), the equation offers us the possibility of prescribing a larger set of vanishing points $\mathcal{S}$, and the set $\mathcal{S} \backslash \mathcal{Z}_{i}$ can be considered as a set of extra sinks. Moreover, the existence of sinks of any kind has a strong influence on the evolution of the solution: thus, $v(x, t)$ must go down to zero as $t \rightarrow \infty$; this is not the case when $\mathcal{S}=\emptyset$ and $v_{0}$ does not go to zero as $|x| \rightarrow \infty$. These results apply of course inside the $\mathcal{C}_{q}$ theory.

WEAK SOLUTIONS. We have found that there are a number of weak solutions that do not fall into our category. Thus, the class of solutions with contracting supports that constitutes a separate theory, cf. [51]. We give some details about these solutions in Section 9. In that section we show examples of solutions with permanent weak zeros, which are not classical solutions, though they are weak. We also construct solutions with permanent singularities, which are forbidden in the $\mathcal{C}_{q}$ theory. Since any such solution has acceptable initial data, the theory developed in this paper provides for an admissible solution in $\mathcal{C}_{q}$ which is smaller than each of these explicit singular solutions and has the same initial data.

Viscosity SOlutions. An option to study this equation is using the modern concept of viscosity solutions, which has been introduced by Crandall and Lions [26], see also [23], for first-order Hamilton-Jacobi equations. The theory has been extended to second-order fully nonlinear equations of elliptic and parabolic type by a number of authors ( $c f$. $[24,17,52])$, to provide a general framework for existence and uniqueness which includes the classical solutions as particular cases. The whole idea of the viscosity theory is defining solutions by testing them with classical super- and sub-solutions via a version of the Maximum Principle. In the case $\gamma<0$ well-posedness of 
the pressure formulation for equation (1.1) is obtained in [18] after a modification of the concept of viscosity supersolution, to include comparison from below with classical free-boundary solutions with expanding supports. But, as already pointed out in [12], in the present situation we are faced with a problem where classical solutions with continuous initial data are not unique, hence the usual viscosity framework cannot provide uniqueness of solutions. The modification performed in [18] cannot be applied since we are not dealing with solutions with expanding supports.

We can restrict the viscosity solutions obtained by the usual definitions (cf. [18] and its references) in the way we did with weak solutions, thus obtaining the class $\mathcal{V}_{s}$ of solutions with constant support and quadratic zeros. Then $\mathcal{V}_{s}=\mathcal{C}_{q}=\mathcal{W}_{s}$ and well-posedness occurs in the sense of Theorems 5.1 and 5.4, so the viscosity concept does not change much. The question of how to obtain well-posedness with a straightforward viscosity definition that could be applied to more general equations of this type (not having a translation into the fast diffusion type) is still a mystery for the authors.

TRACES. In the same line, our concept of $p$-trace is strongly tied to the transformation into fast diffusion, and we do not know how to formulate a concept of trace which would imply well-posedness for other simple equations like

$$
v_{t}=a(x, t, v) \Delta v+b(x, t, v, \nabla v) .
$$

Certainly, each type of equation requires a suitable definition of trace, which reflects how the initial data are seen (or taken) by solutions. Further research into this subject will clarify the theory of degenerate fully-nonlinear parabolic equations.

LOWER RANGE. The methods we develop here cannot be completely extrapolated to the range $0 \leqslant \gamma<N / 2$, because the condition of quadratic zeros loses its relevance. We refer to the works of Bertsch and collaborators quoted above for information on weak solutions and vanishing viscosity, also to $[2,7,51]$ for contracting solutions. For general initial data we have made a detailed comment on the results that are still valid for the fast diffusion formulation in the paper [21]. The subject deserves a detailed study.

Acknowledgments: This work has been finished during a stay of the authors at the University of Texas, Austin. They wish to thank the Department of Mathematics and the TICAM Institute for their hospitality and L. Caffarelli for his interest. A part of the results have first appeared in the Doctoral dissertation [19]. 


\section{References}

[1] Adams, D. R. And Hedberg, L. I.: Function spaces and potential theory. Grundlehren Math. Wissen. 314, Springer Verlag, Berlin, 1996.

[2] Angenent, S. B.: Local existence and regularity for a class of degenerate parabolic equations. Math. Ann. 280 (1988), 465-482.

[3] Aronson, D. G.: The Porous Medium Equation, Some problems of Nonlinear Diffusion. Lectures Notes in Mathematics 1224, Springer-Verlag, New York, 1986.

[4] Aronson, D. G. And BÉnilan, P.: Régularité des solutions de l'équation des milieux poreux dans $\mathbb{R}^{N}$. C. R. Acad. Sci. Paris Sér. I Math. 288 (1979), $103-105$.

[5] Barenblatt, G. I.: On self-similar motion of compressible fluids in porous media. Prikl. Mat. Mekh. 16 (1952), 679-698 (in Russian).

[6] Barenblatt, G. I.: Self-similar intermediate asymptotics for nonlinear parabolic free-boundary problems which occur in image processing. Proc. Natl. Acad. Sci. USA 98 (2001), no. 23, 12878-12881 (electronic).

[7] Barenblatt, G. I., Bertsch, M., Chertock, A. E. and ProstokISHIN, V. M.: Self-similar asymptotics for a degenerate parabolic filtrationabsorption equation. Proc. Natl. Acad. Sci. USA 97 (2000), no. 18, 98449848 (electronic).

[8] Barenblatt, G. I. And VÁzquez, J. L.: Nonlinear Diffusion and Image Enhancement, submitted.

[9] Bénilan, P. and Crandall, M. G.: Regularizing effects of homogeneous evolution equations. In Contribution to Analysis and Geometry (D. N. Clark et al., eds.), 23-30. John Hopkins Univ. Press, Baltimore, Md., 1981.

[10] Berger, M., Gauduchon, P. and Mazet, E.: Le spectre d'une Variété Riemmanienne. Lecture Notes in Mathematics 194, Springer Verlag, Berlin, 1970.

[11] Berryman, J. G. And Holland, C. J.: Stability of the separable solution for fast diffusion equation. Arch. Rat. Mech. Anal. 74 (1980), 379-388.

[12] Bertsch, M. And Ughi, M. Positivity properties of viscosity solutions of a degenerate parabolic equation. Nonlinear Anal. 14 (1990), 571-592.

[13] Bertsch, M., Dal Passo, R. And UGhi, M.: Discontinuous "viscosity" solutions of a degenerate parabolic equation. Trans. Amer. Math. Soc. 320 (1990), 779-798.

[14] Bertsch, M., Dal Passo, R. and UGhi, M.: Nonuniqueness of solutions of a degenerate parabolic equation, Annali Mat. Pura Appl. 161 (1992), $57-81$. 
[15] Blanchard, P., Murat, F. and Redwane, H.: Existence et unicité de la solution renormalisée d'un problème parabolique non linéaire assez général. C. R. Acad. Sci. Paris Sér. I Math. 329, 7 (1999), 575-580. Also, Existence and uniqueness of a renormalized solution for a fairly general class of nonlinear parabolic problems. J. Differential Equations 177 (2001), no. $2,331-374$.

[16] Brezis, H. And Friedman, A.: Nonlinear parabolic equations involving measures as initial conditions. J. Math. Pures Appl. (9) 62 (1983), 73-97.

[17] Caffarelli, L. A. And Cabré, X.: Fully nonlinear elliptic equations. Coll. Publ. 43, Amer. Math. Soc., Providence, 1995.

[18] Caffarelli, L. A. And VÁzquez, J. L.: Viscosity solutions for the porous medium equation. In Differential equations: La Pietra 1996 (Florence), 1326. Proc. Sympos. Pure Math. 65, Amer. Math. Soc., Providence, RI, 1999.

[19] Chasseigne, E.: Thesis, Univ. Tours, France. December 2001.

[20] Chasseigne, E.: Classification of Razor Blades to the filtration equation. The sub-linear case. J. Differential Equations 187 (2003), 72-105.

[21] Chasseigne, E. And VÁzquez, J. L.: Extended theory of fast diffusion equations in optimal classes of data. Radiation from singularities. Arch. Rat. Mech. Anal. 164 (2002), 133-187.

[22] Chasseigne, E. And VÁzquez, J. L.: Weak Solutions of Fast Diffusion Equations in bounded domains, submitted.

[23] Crandall, M. G., Evans, L. C. and Lions, P. L.: Some properties of viscosity solutions of Hamilton-Jacobi equations. Trans. Amer. Math. Soc. 282 (1984), 487-502.

[24] Crandall, M. G., Ishit, H. and Lions, P. L.: User's guide to viscosity solutions for second-order partial differential equations. Bull. Amer. Math. Soc. 27 (1992), 1-67.

[25] Crandall, M. G. and Lions, P. L.: Condition d'unicité pour les solutions généralisées des équations de Hamilton-Jacobi du premier ordre. (French. English summary) C. R. Acad. Sci. Paris Sér. I Math. 292 (1981), no. 3, 183-186.

[26] Crandall, M. G. And Lions, P. L.: Viscosity solutions of HamiltonJacobi equations. Trans. Amer. Math. Soc. 277 (1983), 1-42.

[27] Dahlberg, B. E. J., Fabes, E. and Kenig, C.: A Fatou theorem for solutions of the Porous Medium Equation. Proc. Amer. Math. Soc. 91 (1984), 205-212.

[28] Dal Masso, G., Murat, F., Orsina, L. and Prignet, A.: Definition and existence of renormalized solution of elliptic equations with general measure data. C. R. Acad. Sci. Paris Sér. I Math. 325 (1997), 5, 481-486. 
[29] Dal Passo, R. and Luckhaus, S.: A degenerate diffusion problem not in divergence form. J. Differential Equations 69 (1987), no. 1, 1-14.

[30] Esteban, J. R., Rodríguez, A. And Vázquez, J. L.: A nonlinear heat equation with singular diffusivity. Comm. Partial Differential Equations 13 (1988), 985-1039.

[31] Esteban, J.R., Rodríguez, A. And Vázquez, J. L.: The maximal solution of the logarithmic fast diffusion equation in two space dimension. Adv. Differential Equations 2 (1997), 867-894.

[32] Evans, L. C. And Gariepy, R. F.: Measure theory and fine properties of functions. Studies in Advanced Mathematics, CRC Press, Boca Raton, FL, 1992.

[33] Friedman, A.: Partial Differential Equations of Parabolic Type. PrenticeHall, Inc., Englewood Cliffs, N.J., 1964.

[34] Friedman, A. And Kamin, S.: The asymptotic behaviour of a gas in an n-dimensional porous medium. Trans. Amer. Math. Soc. 262 (1980), $373-401$.

[35] Galaktionov, V. A., Peletier, L. A. and Vázquez, J. L.: Asymptotics of the fast diffusion equation with critical exponent. SIAM J. Math. Anal. 31 (2000), no 5, 1157-1174.

[36] Herrero, M. A. And Pierre, M.: The Cauchy problem for $u_{t}=\Delta u^{m}$ when $0<m<1$. Trans. Amer. Math. Soc. 291 (1985), 145-158.

[37] Hui, K. M.: Fatou theorem for the solutions of some nonlinear equations. J. Math. Anal. Appl. 183 (1994), 37-52.

[38] Kamin, S. And Dascal, L.: Long time slow expansion of hot bubbles in gases. In Optimal Control and Partial Differential Equations. J.L. Menaldi et al. (eds), IOS Press, 2001.

[39] KING, J. R.: Self-similar behaviour for the equation of fast nonlinear diffusion. Phil. Trans. Roy. Soc. London A 343 (1993), 337-375.

[40] King, J. R.: Exact polynomial solutions to some nonlinear diffusion equations. Phys. D 64 (1993), no. 1-3, 35-65.

[41] Ladyzhenskaya, O. A., Solonnikov, V. A. and Ural'tseva, N. N.: Linear and Quasilinear Equations of Parabolic Type. Transl. Math. Monographs 23, Amer. Math. Soc, Providence, 1968.

[42] Marcus, M. And VÉron, L.: Initial trace of positive solutions of some nonlinear parabolic equations. Comm. Partial Differential Equations 24 (1999), 1445-1499.

[43] Meerson, B.: On the dynamics of strong temperature disturbances in the upper atmosphere of the Earth. Phys. Fluids A 1 (1989), 887-891.

[44] Meerson, B., Sasorov, P. V. and Sekimoto, K.: Logarithmically slow expansion of hot bubbles in gases. Phys. Rev. E 61 (2000), 1403-1406. 
[45] Peletier, M. A. And Zhang, H. F.: Self-similar solutions of fast diffusion equations that do not conserve mass. Differential Integral Equations 8 (1995), 2045-2064.

[46] Rodríguez, A. And VÁzquez, J. L.: Nonuniqueness of solutions of nonlinear heat equations of fast diffusion type. Ann. Inst. H. Poincaré, Anal. non Linéaire 12 (1995), 173-200.

[47] UGHI, M.: A degenerate parabolic equation modelling the spread of an epidemic. Ann. Mat. Pura Appl. (4) 143 (1986), 385-400.

[48] VÁzquez, J. L.: Behaviour of the velocity of one-dimensional flows in porous media. Trans Amer. Mat. Soc. 286 (1984), 787-802.

[49] VÁzquez, J. L.: Nonexistence of solutions for nonlinear heat equations of fast-diffusion type. J. Math. Pures Appl. 71 (1992), 503-526.

[50] VÁzquez, J. L.: An introduction to the mathematical theory of the porous medium equation. In Shape optimization and free boundaries (Montreal, PQ, 1990), 347-389. NATO Adv. Sci. Inst. Ser. C Math. Phys. Sci. 380. Kluwer Acad. Publ., Dordrecht, 1992.

[51] VÁzquez, J. L.: Darcy's Law and the theory of shrinking solutions of fast diffusion equations. To appear in SIAM J. Math. Anal.

[52] WANG, L. H.: On the regularity theory of fully nonlinear parabolic equations: I. Comm. Pure Applied Math. 45 (1992), no 1, 27-76. On the regularity theory of fully nonlinear parabolic equations: II. Comm. Pure Applied Math. 45 (1992), no 2, 141-178.

Recibido: 7 de junio de 2001

Emmanuel Chasseigne Laboratoire de Mathématiques et Physique Théorique,

Université de Tours Parc de Grandmont, 37200 Tours, France. echasseigne@univ-tours.fr

Juan Luis Vázquez Departamento de Matemáticas, Universidad Autónoma de Madrid 28049 Madrid, Spain. juanluis.vazquez@uam.es 